\title{
ROS-Mediated Apoptosis and Autophagy in Ovarian Cancer Cells Treated with Peanut-Shaped Gold Nanoparticles
}

This article was published in the following Dove Press journal: International Journal of Nanomedicine

\author{
Ewelina Piktel (D) \\ Ilona Ościłowska iD ${ }^{2}$ \\ Łukasz Suprewicz (iD) \\ Joanna Depciuch iD ${ }^{3}$ \\ Natalia Marcińczyk ${ }^{4}$ \\ Ewa Chabielska ${ }^{4}$ \\ Przemysław Wolak (iD ${ }^{5}$ \\ Tomasz Wollny ${ }^{6}$ \\ Marianna Janion ${ }^{5}$ \\ Magdalena Parlinska- \\ Wojtan (iD ${ }^{3}$ \\ Robert Bucki (iD) \\ 'Department of Medical Microbiology and \\ Nanobiomedical Engineering, Medical \\ University of Bialystok, Bialystok, 15-222, \\ Poland; ${ }^{2}$ Department of Medicinal \\ Chemistry, Medical University of Bialystok, \\ Bialystok, I5-222, Poland; ${ }^{3}$ Institute of \\ Nuclear Physics Polish Academy of \\ Sciences, Krakow, PL-31342, Poland; \\ ${ }^{4}$ Department of Biopharmacy, Medical \\ University of Bialystok, Bialystok, 15-222, \\ Poland; ${ }^{5}$ Institute of Medical Sciences, \\ Collegium Medicum, Jan Kochanowski \\ University, Kielce, 25-317, Poland; ${ }^{6} \mathrm{Holy}$ \\ Cross Cancer Center in Kielce, Kielce, 25- \\ 734, Poland
}

Correspondence: Robert Bucki

Department of Medical Microbiology and

Nanobiomedical Engineering, Medical

University of Bialystok, Mickiewicza 2C.

Bialystok, 15-222, Poland

Tel + 48857485793

Email buckirobert@gmail.com
Background: Even with considerable improvement in treatment of epithelial ovarian cancer achieved in recent years, an increasing chemotherapy resistance and disease 5-year relapse is recorded for a majority part of patients that encourages the search for better therapeutic options. Gold nanoparticles (Au NPs) due to plethora of unique physiochemical features are thoroughly tested as drug delivery, radiosensitizers, as well as photothermal and photodynamic therapy agents. Importantly, due to highly controlled synthesis, it is possible to obtain nanomaterials with directed size and shape.

Methods: In this work, we developed novel elongated-type gold nanoparticles in the shape of nanopeanuts (AuP NPs) and investigated their cytotoxic potential against ovarian cancer cells SKOV-3 using colorimetric and fluorimetric methods, Western blot, flow cytometry, and fluorescence microscopy.

Results: Peanut-shaped gold nanoparticles showed high anti-cancer activity in vitro against SKOV-3 cells at doses of $1-5 \mathrm{ng} / \mathrm{mL}$ upon 72 hours treatment. We demonstrate that AuP NPs decrease the viability and proliferation capability of ovarian cancer cells by triggering cell apoptosis and autophagy, as evidenced by flow cytometry and Western blot analyses. The overproduction of reactive oxygen species (ROS) was noted to be a critical mediator of AuP NPs-mediated cell death.

Conclusion: These data indicate that gold nanopeanuts might be developed as nanotherapeutics against ovarian cancer.

Keywords: gold nanoparticles, ovarian cancer, gold nanopeanuts, anti-cancer therapy, nanotechnology, apoptosis, autophagy

\section{Introduction}

Epithelial ovarian cancer (EOC) derived from malignant transformation of the ovarian epithelium, peritoneum or fallopian tube, is recognized as one of the deadliest malignancies of women. Due to the non-specificity of the early signs of EOC, as well as the lack of effective screening methods, in most patients the tumor is diagnosed at an advanced stage of development, which not only significantly worsens the clinical outcome, but also considerably limits the therapeutic options. ${ }^{1}$ Currently, the main therapeutic procedure includes cytoreductive surgery in combination with chemotherapy. ${ }^{1,2}$ Optimization of the carboplatin-paclitaxel scheme as first-line combined chemotherapy followed by intraperitoneal administration of cytostatics contributed to significant progress in the treatment of patients with advanced ovarian cancer. ${ }^{3}$ Unfortunately, despite an improvement in the clinical 
response and an increase in the average survival, $60-80 \%$ of patients still experienced ineffectiveness of the therapy used and disease relapse within 5 years, which is due to the resistance of tumors to the cytostatic treatment. ${ }^{4}$ Therefore, the search for new therapeutic methods effective in the treatment of ovarian cancers is particularly urgent and clinically relevant.

The rapid development of nanotechnology techniques observed in recent years has created the possibility of using nanomaterials in modern cancer therapy. ${ }^{5}$ Among them, metallic nanoparticles, including gold nanoparticles (Au NPs) have aroused considerable interest. ${ }^{6}$ Optimized protocols for the synthesis of varied-shaped Au NPs with adjusted size, as well as unique physicochemical properties of nanogold, ease of surface functionalization, controllable biodistribution and satisfactory biocompatibility broadens the range of their potential biomedical applications, with particular emphasis on cancer treatment. ${ }^{6,7}$ In effect, a growing number of reports demonstrate the utility of Au NPs as drug carriers, ${ }^{8}$ components of photothermal therapy, ${ }^{9}$ contrast factors, ${ }^{10}$ and therapeutic agents with significant cytotoxic activity, ${ }^{11}$ which highlights the clinical potential of Au NPs as the basis of innovative therapies against breast cancer, ${ }^{12}$ gastrointestinal tract cancers, ${ }^{13}$ lung cancer, ${ }^{14}$ and ovarian cancer. ${ }^{11}$ With respect to ovarian cancer the proposed mechanism of anticancer action involves the formation of reactive oxygen species in the cell, entry of the cell into uncompensated state of oxidative stress, damage to genetic material and biological membranes, and initiation of apoptosis. ${ }^{11}$ Other studies also demonstrate the ability of gold nanoparticles to inhibit angiogenesis and metastases in a mouse model of ovarian cancer, ${ }^{15}$ as well as the impact on secretory cytokines profile. ${ }^{16}$ It is important, however, that the vast majority of scientific research was conducted using spherical-shaped nanoparticles. A number of animal experiments focusing on the assessment of the therapeutic potential and pharmacokinetic parameters of metal nanoparticles in vivo have confirmed that the physicochemical properties of nanomaterials, particularly size and shape, significantly affect intravascular transport processes, retention in cancer tissues, and antitumor efficacy. ${ }^{17,18}$ To date, it was demonstrated that non-spherical nanoparticles show a higher accumulation in cancerous tumors and thus higher therapeutic efficacy compared to spherical nanomaterials of the same structure, dose, and comparable other physicochemical parameters. ${ }^{19}$ The change in the shape of nanoparticles from spherical to stick-like (eg, to nanorods) also improves the ability to absorb radiation, improving the effectiveness of photothermal therapy. ${ }^{20}$

Here we investigated the cytotoxic activity and mechanism of action of newly-developed peanut-shaped gold nanoparticles (AuP NPs) against epithelial ovarian cancer cells SKOV-3. The peanut-like shape of the Au NPs was designed with an attempt to obtain a larger active surface and to keep and potentially to decrease their cytotoxicity to a level comparable with the spherical one. Therefore, we believe that from the perspective of biomedical application, the properties of our nanoparticles are the most significant advantage in comparison with the commonly studied Au NPs with spherical shape. According to our best knowledge, it is the first study demonstrating anti-cancer activity of gold nanopeanuts, and a first study deeping the mechanism of anti-cancer activity of these unreported to date, newly-shaped nanomaterials. In presented work we demonstrate that gold nanoparticles induced apoptosis and autophagy through the modulation of the ROS/JNK signaling pathway in human ovarian cancer cells SKOV-3. The presented results may contribute to develop more effective therapies for human ovarian cancer treatment.

\section{Materials and Methods \\ Materials}

Reagents for gold nanoparticles synthesis (cetrimonium bromide $[\mathrm{CTAB}]$, gold (III) chloride hydrate $\left[\mathrm{HAuCl}_{4}\right]$, silver nitrate $\left[\mathrm{AgNO}_{3}\right]$, sodium borohydride $\left[\mathrm{NaBH}_{4}\right]$, ascorbic acid $\left.\left[\mathrm{C}_{6} \mathrm{H}_{8} \mathrm{O}_{6}\right]\right)$ and all other required chemicals were ordered from Sigma-Aldrich (Saint Louis, MO, USA). Human ovarian adenocarcinoma cells SKOV-3 (ATCC ${ }^{\circledR}$ HTB-77' ${ }^{\mathrm{TM}}$ ) were from American Type Culture Collection (ATCC, Manassas, VA, USA). Dulbecco's Modified Eagle's Medium (DMEM) supplemented with $4 \mathrm{mM}$ L-glutamine, $4500 \mathrm{mg} / \mathrm{L}$ glucose, 1 $\mathrm{mM}$ sodium pyruvate, and $1,500 \mathrm{mg} / \mathrm{L}$ sodium bicarbonate was from ATCC. Phosphate-buffered saline (PBS), TrypsinEDTA Solution 10×, and Antibiotic Antimycotic Solution $100 \times$ (with 10,000 units penicillin, $10 \mathrm{mg}$ streptomycin, and $25 \mu \mathrm{g}$ amphotericin B per $\mathrm{mL}$ ) were purchased from SigmaAldrich. MTT salt (3-(4,5-Dimethyl-2-thiazolyl)-2,5-diphenyl-2H-tetrazolium bromide), Neutral red, and $2^{\prime}, 7^{\prime}$ dichlorofluorescin diacetate (DFCH-DA) were from SigmaAldrich. The CyQUANT Cell Proliferation Assay Kit was ordered from Invitrogen (Thermo Fisher Scientific, Waltham, MA, USA). Reagents for intracellular GSH and mitochondrial potential quantification: Solution 5 (VB-48 $\left.{ }^{\mathrm{TM}} / \mathrm{PI} / \mathrm{AO}\right)$, 
Solution $7(200 \mu \mathrm{g} / \mathrm{mL}$ JC-1) and Solution $8(1 \mu \mathrm{g} / \mathrm{mL}$ DAPI in PBS) were from Chemometec (Allerod, Denmark). Flow cytometry assay kits: Muse ${ }^{\circledR}$ Count \& Viability Kit and Muse $^{\circledR}$ Bcl-2 Activation Dual Detection Kit were produced by Luminex Corporation (Austin, TX, USA). Primary and secondary anti-apoptotic and anti-autophagy antibodies were from Cell Signaling Technology (CS, Danvers, MA, USA), Abcam (Cambridge, UK), and Sigma-Aldrich. The following antibodies were used in this study: rabbit anti-NADPH oxidase 4 [UOTR1B493] antibody (NOX4) (Abcam, cat. no: ab133303), rabbit anti-annexin A1 (D5V2T) antibody (CS, cat. no: 32,934), rabbit anti-cleaved-poly (ADP-ribose) polymerase-1 (PARP) (Asp214) antibody (CS, cat. no: 5625), rabbit anti-cleaved-caspase-3 (Asp175) antibody (CS, cat. no: 9664), rabbit anti-cleaved-caspase-9 (Asp330) antibody (CS, cat. No: 7237), rabbit anti-COX IV (3E11) antibody (CS, cat. no: 4850), rabbit anti-LC3A/B (D3U4C) antibody (CS, cat. no: 12,741), rabbit anti-Beclin-1 (D40C5) antibody (CS, cat. no: 3495), rabbit anti-Atg12 (D88H11) antibody (CS, cat. no: 4180), rabbit anti-Atg7 (D13B11) antibody (CS; cat. no: 8558$)$, and monoclonal anti- $\beta$-Actin antibody produced in mouse (Sigma Aldrich, cat. no: A2228). Secondary HRPlinked, Alexa Fluor 488-conjugated and FITC-conjugated antibodies were from Sigma Aldrich, Abcam, and Becton, Dickinson \& Company, respectively. 5\% non-fat dry milk was from BioRad (Hercules, CA, USA). Amersham ECL Western Blotting Detection Reagent was purchased from GE Healthcare Life Sciences (Little Chalfont, Buckinghamshire, UK). JNK inhibitor II (SP600125), apoptosis inhibitor (Z-VAD-FMK) and autophagy inhibitor (3-methyladenine, 3-MA) were from Sigma-Aldrich.

\section{Synthesis and Physicochemical Characterization of Peanut-Shaped Nanoparticles (AuP NPs)}

The synthesis of AuP NPs consists of two reactions. First, $5 \mathrm{~mL}$ of $10^{-3} \mathrm{M}$ CTAB was prepared. Next, $5 \mathrm{~mL}$ of $5 \times 10^{-4} \mathrm{M} \mathrm{HAuCl}_{4}$ and $0.6 \mathrm{~mL}$ of $100 \times 10^{-3} \mathrm{M} \mathrm{NaBH}_{4}$ was added to this solution. The solution was permanently under vigorous stirring. The reaction was stopped, when the solution color changed to red. In the second step, $5 \mathrm{~mL}$ of $10^{-3} \mathrm{M}$ CTAB was prepared. Next, $0.2 \mathrm{~mL}$ of $3.97 \times 10^{-2}$ $\mathrm{M} \mathrm{AgNO}, 5 \mathrm{~mL}$ of $5 \times 10^{-4} \mathrm{M} \mathrm{HAuCl} 4,70 \mu \mathrm{L}$ of $7.86 \times 10^{-2} \mathrm{C}_{6} \mathrm{H}_{8} \mathrm{O}_{6}$, and $30 \mu \mathrm{L}$ of $\mathrm{Au}$ nanoseeds were added to the solution. The solution was permanently under vigorous stirring and the reaction was stopped 3 hours after change solution color for red.
The morphology of obtained AuP NPs was examined by scanning transmission electron microscopy (STEM) using the high-angle annular dark field detector (HAADF), in conventional and high-resolution mode. All these measurements were performed on an aberrationcorrected FEI Titan electron microscope operating at 300 $\mathrm{kV}$ equipped with a FEG cathode. The particle size distribution was evaluated based on the HRSTEM images taken from different areas of the TEM grids. For each sample, the diameter of 100 nanoparticles was measured. The zeta potential distribution of AuP NPs was determined by the microelectrophoretic method using Zetasizer Nano Series from Malvern Instruments. The Smoluchowski model was used in zeta potential measurements. Each value was obtained as an average of three subsequent runs of the instrument with at least 20 measurements. All experiments were performed in water at $25^{\circ} \mathrm{C}$. Lambda Bio20 instrument from Perkin Elmer was used to measure the Ultraviolet-visible UV-Vis spectra of the synthesized gold nanoparticles. The scan speed was $240 \mathrm{~nm} / \mathrm{min}$ and the resolution was $1 \mathrm{~nm}$. In this experiment, the used spectral range was from $200 \mathrm{~nm}$ to $900 \mathrm{~nm}$.

\section{Cell Culture}

Human ovarian adenocarcinoma cells SKOV-3 (ATCC ${ }^{\circledR}$ HTB-77 ${ }^{\mathrm{TM}}$ ) were cultured in high-glucose DMEM supplemented with $10 \%$ fetal bovine serum (FBS), glutamine (2 $\mathrm{mM} / \mathrm{L})$, penicillin $(50 \mathrm{U} / \mathrm{mL})$, and streptomycin $(50 \mu \mathrm{g} /$ $\mathrm{mL}$ ) and maintained at $37^{\circ} \mathrm{C}$ in an atmosphere containing $5 \% \mathrm{CO}_{2}$ with saturated humidity. For experiments, cells were seeded at a density of $1 \times 10^{4}$ or $5 \times 10^{4}$ cells/well (for 96-well plates and 24-well plates, respectively) and cultured with AuP NPs for 24, 48, or 72 hours (for cytotoxicity evaluation), 24 hours (for experiments involving signaling pathways inhibitors), or 72 hours exclusively (for mechanism determination assays).

\section{Cytotoxicity Assessment}

Anti-cancer activity of tested nanoparticles against ovarian adenocarcinoma was explored by measurement of metabolic activity of treated cancer cells, estimation of alterations in DNA biosynthesis, and investigation of membrane permeability of ovarian cancer cells.

Initially, viability of treated cancer cells was measured using MTT assay, as described previously. ${ }^{21}$ Every 24 hours, cells were washed twice with PBS followed by addition of MTT working solution at a final concentration 
of $0.5 \mathrm{mg} / \mathrm{mL}$ and further incubated for 4 hours. Formed formazan precipitate was dissolved using DMSO and absorbance was recorded using $540 \mathrm{~nm}$ wavelength (Varioskan Lux, Thermo Fisher Scientific, Waltham, MA, USA). Cancer cells survival was calculated as a percentage when compared to control $(0 \mu \mathrm{g} / \mathrm{mL}$ of AuP NPs, $100 \%$ survival).

To assess the impact of AuP NPs on proliferation capability and DNA biosynthesis in treated SKOV-3 cells, CyQUANT Cell Proliferation Assay Kit was used according to manufacturer's guidelines. At the indicated times, the culture medium from AuP NPs-treated cells was discarded, cells were washed with PBS, and plates were frozen. Next, cells were lysed using CyQUANT dye mix, and total cellular nucleic acid was measured by fluorometer at $480 / 520 \mathrm{~nm}$ wavelengths (VICTOR $^{\text {TM }}$ X4 Multilabel Plate Reader, PerkinElmer, MA, USA).

Neutral red uptake (NRU) assay, performed according to the protocol by Borenfreund and Puerner $^{22}$ was employed to elucidate the permeability of treated cancer cells upon AuP NPs-mediated treatment. At indicated time-points, the culture medium was removed, cells were washed with PBS, and a $100 \mu \mathrm{L} /$ well of Neutral Red solution $(50 \mu \mathrm{g} / \mathrm{mL})$ was added. After 30 minutes, the cells were washed with PBS and dye from viable cells was released by extraction with a mixture of acetic acid, ethanol, and water $(1 \% / 50 \% / 49 \%$, respectively). After 10 minutes of shaking, the absorbance of the dissolved Neutral Red was measured at $540 \mathrm{~nm}$ in a microplate reader using a blank as a reference. Cytotoxicity was expressed as a percentage of the control $(0 \mu \mathrm{g} / \mathrm{mL}, 100 \%$ of intact membranes).

To confirm the results from cytotoxicity assessments, cells after 72 hours incubation with gold nanopeanuts were inspected for alterations in morphological features using light microscopy at 40x magnification. Viability of SKOV3 cells upon 72 hours treatment with AuP NPs (0.5-5 ng/ $\mathrm{mL}$ ) was also confirmed by flow cytometry using Muse ${ }^{\circledR}$ Count \& Viability Kit.

\section{Analysis of the Level of Intracellular GSH}

To examine the hypothesis that the cytotoxic effect of developed gold nanopeanuts is determined by disruption of oxidative balance in treated cancer cells, the amount of free thiols in AuP NPs-treated was estimated using staining with VitaBright-48 ${ }^{\mathrm{TM}}$ (VB-48 ${ }^{\mathrm{TM}}$ ), non-fluorescent compound reacting with cellular thiols forming a fluorescent product. To quantify the level of reduced glutathione (GSH), SKOV-3 cells were treated with AuP NPs at concentrations of $0.5,1$, and $5 \mathrm{ng} / \mathrm{mL}$ for 72 hours, washed twice with sterile PBS, harvested, and incubated with Solution 5 consisting of VB- $48^{\mathrm{TM}}$, propidium iodide, and acridine orange mixture for 5 minutes prior analysis using NucleoCounter ${ }^{\circledR} \quad$ NC-3000 ${ }^{\mathrm{TM}} \quad$ (Chemometek, Allerod, Denmark).

\section{ROS Generation Assessment}

Intracellular reactive oxygen species accumulation was measured using DCFH-DA as a fluorescent probe. Briefly, cultured SKOV-3 cells were pre-incubated with DCFH-DA $(20 \mu \mathrm{M})$ in culture medium for 30 minutes, washed twice with PBS, and treated with increasing concentrations of AuP NPs up to 72 hours. Every 24 hours a fluorescent intensity was measured at excitation/emission wavelength of 488/535 nm using a Varioskan Lux microplate reader and represented by a fold-increase compared with control cells.

\section{Detection of Mitochondrial Potential Disruption}

The AuP NPs-induced alterations in mitochondrial potential of ovarian cancer cells were measured using the NucleoCounter ${ }^{\circledR}$ NC-3000TM fluorescence image cytometer. For this purpose, SKOV-3 cells treated with AuP NPs were incubated with Solution 7 (at final JC-1 concentration of $2.5 \mu \mathrm{g} / \mathrm{mL}$ ) for 10 minutes at $37^{\circ} \mathrm{C}$, washed thoroughly with PBS, resuspended in Solution $8(1 \mu \mathrm{g} /$ $\mathrm{mL}$ DAPI in PBS) and analyzed immediately by NucleoCounter ${ }^{\circledR}$ NC-3000 ${ }^{\text {TM }}$ software.

\section{Immunofluorescence Staining}

Accumulation and expression of apoptotic and autophagicrelated proteins was visualized by immunostaining method. Briefly, SKOV-3 cells grown on coverslip were treated with AuP NPs for 72 hours, fixed with 4\% formaldehyde diluted in PBS, permeabilized with $0.1 \%$ Triton X-100 (10 minutes), and blocked for 30 minutes with blocking buffer $[1 \%$ bovine serum albumin (BSA), glycine $(22.5 \mathrm{mg} / \mathrm{mL})$ diluted in TBS-T (PBS supplemented with $0.1 \%$ Tween-20)]. After blocking cells were incubated (1 hour, room temperature) with monoclonal primary antibodies against NADPH oxidase 4 (NOX4), annexin A1, cleaved PARP, cleaved caspase-3, cleaved caspase-9, COX-IV, LC3A/B, Beclin-1, Atg12, and Atg7. Next, cells were washed three times with PBS (3 minutes) 
and incubated with Alexa Fluor 488-conjugated goat antirabbit secondary antibody (for NOX4 and ANXA1 staining) or FITC goat anti-mouse IgG and anti-rabbit IgG antibodies for 1 hour in the dark. Cells were washed with PBS (3x3 minutes), incubated with Hoechst 33,342 for 15 minutes to counterstain, if necessary, and analyzed using a confocal laser scanning microscope.

\section{Bcl-2 Activity Investigation}

Measurement of total levels of Bcl-2 expression in ovarian cancer cells treated with developed nanopeanuts was carried out using the Muse ${ }^{\mathrm{TM}}$ Bcl-2 Activation Dual Detection Kit as guided by the manufacturer. Briefly, AuP NPs-treated cells after 72 hours incubation were washed with PBS, harvested, and fixed for 5 minutes on ice. After rinsing with PBS, cells were permeabilized with permeabilization buffer for 5 minutes on ice followed by washing and incubation of cells with antibody cocktail for 30 minutes at room temperature in the dark. Samples were analyzed using a Muse ${ }^{\mathrm{TM}}$ Cell Analyzer (Luminex Corporation, Austin, TX, USA).

\section{Multicaspase Assay}

Pan Caspase assay (Muse ${ }^{\mathrm{TM}}$ MultiCaspase Kit), detecting the presence and activation of multiple caspases, including caspase-1, 3, 4, 5, 6, 7, 8, and 9, was employed to investigate whether the cytotoxic effect of gold nanoparticles is dependent on caspases activity. For this purpose, AuP NPs-treated cells were harvested, washed, and incubated with Multicaspase working solution for 30 minutes at $37^{\circ}$ C. To distinguish cells with membrane permeable, 7-actinomycin D (7-AAD) solution was added, and cells were analyzed using Muse ${ }^{\mathrm{TM}}$ Cell Analyzer.

\section{Western Blot Analysis}

To quantitively elucidate the potential signaling pathways altered by gold nanoparticles treatment, Western blot analyses were carried out. To perform this, SKOV-3 cells were cultured for 72 hours with different concentrations of AuP NPs. The Laemmli method was used to perform SDS-PAGE electrophoresis. ${ }^{23}$ Equal amounts of cell supernatants (30 mg of protein) were analyzed. SKOV-3 cell lysates were harvested and subjected to SDS-PAGE in $10 \%$ polyacrylamide gel electrophoresis for 1 hour, at room temperature $(125 \mathrm{~V})$. The protein was transferred to $0.2 \mu \mathrm{m}$ pore-sized nitrocellulose (wet transfer, 1 hour, $100 \mathrm{~mA}, \mathrm{RT}$ ) and then blocked with 5\% non-fat dry milk in TBS-T $(20 \mathrm{mmol} / \mathrm{L}$ Tris- $\mathrm{HCl}, 150 \mathrm{mmol} / \mathrm{L}$ $\mathrm{NaCl}, 0.05 \%$ Tween 20 , adjusted to $\mathrm{pH} 7.4$ ) and incubated with the following primary monoclonal antibodies: anti- $\beta$-actin, anti-cleaved-PARP, anti-cleaved Caspase-3, anti-cleaved Caspase-9, and anti-COX IV (for apoptosis evaluation) or antiLC3A/B, anti-Beclin-1, anti-Atg12, and anti-Atg-7 (for autophagy evaluation) antibodies, diluted 1:1,000 in blocking buffer overnight at $4{ }^{\circ} \mathrm{C}$. After incubation with primary antibodies, the membranes were washed in TBS with $0.05 \%$ Tween (TBST) three times for 15 minutes and incubated with anti-goat, antirabbit, or anti-mouse HRP-linked secondary antibody at concentration 1:7,500 for 60 minutes at room temperature with gentle agitation. After washing in TBS-T ( $3 \times 5$ minutes) membranes were incubated with Amersham ECL Western Blotting Detection Reagent. Pictures were taken using BioSpectrum Imaging System UVP (Ultra-Violet Products Ltd, Cambridge, UK). Densitometry of Western blot bands was analyzed using ImageJ software.

\section{Evaluation of Biological Activity of Gold Nanopeanuts Using Signaling Pathways Inhibitor}

To examine the interrelationships between observed effects, SKOV-3 cells were pre-treated for 2 hours with JNK inhibitor II (SP600125; $10 \mu \mathrm{M})$, apoptosis inhibitor (Z-VAD-FMK; $20 \mu \mathrm{M}$ ), or 3-methyladenine (3-MA; $5 \mathrm{mM}$ ), washed with PBS, exposed to AuP NPs at concentrations of $0.5,1$, or $5 \mathrm{ng} / \mathrm{mL}$ for 24 hours and analyzed according to the above-described procedures.

\section{Statistical Analysis}

The provided data are results from three independent experiments \pm SD. The significance of differences was determined using the two-tailed Student's $t$-test. Statistical analyses were performed using OriginPro 2020 (OriginLab Corporation, Northampton, USA). $P<0.05$ was considered to be statistically significant.

\section{Results \\ Physicochemical Analysis of Peanut-Shaped Gold Nanoparticles}

STEM and HRSTEM images of AuP NPs showed that synthesized nanoparticles are characterized by a peanut shape and have a crystalline structure (Figure 1A). The size of obtained NPs along the longitudinal axis is $60.00 \pm 4.24 \mathrm{~nm}$, while along the transverse axis it is $30 \pm 3.49 \mathrm{~nm}$ (Figure 1B). Consequently, the size spread of the AuP NPs is negligible. The zeta potential values of AuP NPs dispersions as a function of $\mathrm{pH}$ are presented in Figure 1C. The AuP NPs are positively 
charged in the entire range of $\mathrm{pH}$ and their zeta potential values range from about 42 for $\mathrm{pH}=3.5$ to $19 \mathrm{mV}$ for $\mathrm{pH}=12.5$. Furthermore, the ultraviolet-visible (UV-Vis) spectrum (Figure 1D) showed that the position of localized plasmon resonances of the obtained AuP NPs was observed at $775 \mathrm{~nm}$.

In order to conclude on biological properties of developed gold nanopeanuts as accurately as possible, we additionally synthetized a new set of spherical gold nanoparticles (AuSph NPs) using CTAB as one of the reagents. The physicochemical characterization of these nanostructures is demonstrated as Supplementary Figure 1. Although the synthesized spherical AuNPs have a size range from 4-12 $\mathrm{nm}$ with an average size of $8 \mathrm{~nm}$, ie, are smaller than tested AuP NPs, both AuSph NPs and AuP NPs are characterized by comparable chemical properties. Similarly, the AuP NPs are characterized by crystalline structure and are positively charged in the entire range of $\mathrm{pH}$.

\section{Peanut-Shaped Gold Nanoparticles Exert Potent Cytotoxic Effect Against Ovarian Cancer Cells at Nanogram Doses}

To determine the inhibitory effects of gold nanopeanuts, ovarian adenocarcinoma cells were treated with various concentrations of AuP NPs for 24, 48, and 72 hours followed by investigation of metabolic activity, the ability to proliferate, and permeability of cellular membranes. As shown in Figure 2A, gold nanoparticles inhibited cell viability in a dose- and time-dependent manner when compared to the control group, with $50 \%$ growth inhibition (IC50) at a concentration of $1.75 \pm 0.03$, $1.20 \pm 0.11$, and $1.03 \pm 0.19 \mathrm{ng} / \mathrm{mL}$ for 24,48 , and 72 hour treatments, respectively. In effect, treatment with AuP NPs at a dose of $1 \mathrm{ng} / \mathrm{mL}$ for 72 hours resulted in SKOV-3 cells viability decreasing to $47.64 \pm 8.52 \%$, and at concentration of $5 \mathrm{ng} / \mathrm{mL}$ no detectable metabolic activity was noted. To compare the anti-cancer activity of peanut-shaped gold nanoparticles with conventional cytostatics used in ovarian cancer therapy, we investigated cytotoxicity of cisplatin, paclitaxel, and doxorubicin using MTT assay and assessed IC50 values for these compounds upon 72 hours incubation as well. As presented in Supplementary Table 1, developed gold nanoparticles are far more effective than conventional cytostatic, suggesting their great potential in development of the new treatment methods against this malignancy.
A
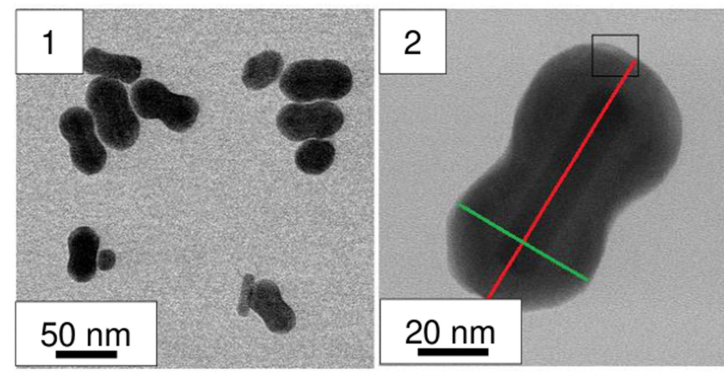

C

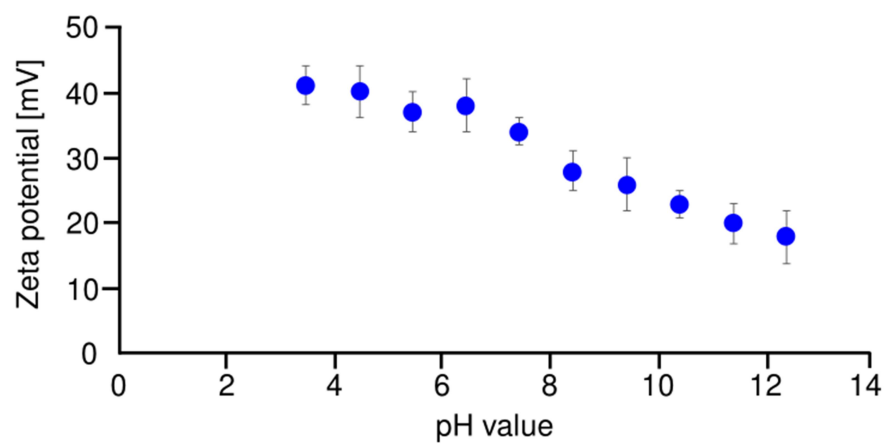

B

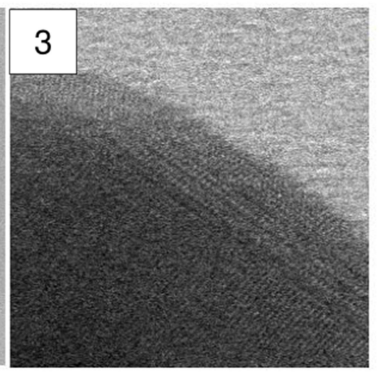

D

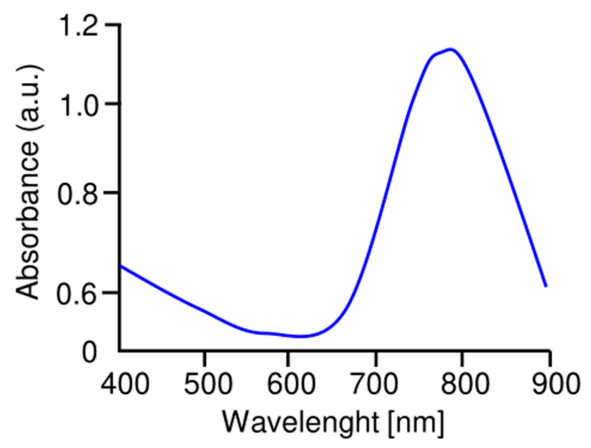

Figure I Physicochemical characterization of peanut-shaped gold nanoparticles. Overview BF STEM (AI and A2) and HRSTEM (A3). AuP NPs size distribution (B), zeta potential in different $\mathrm{pH}(\mathbf{C})$ and UV-Vis spectrum (D). 

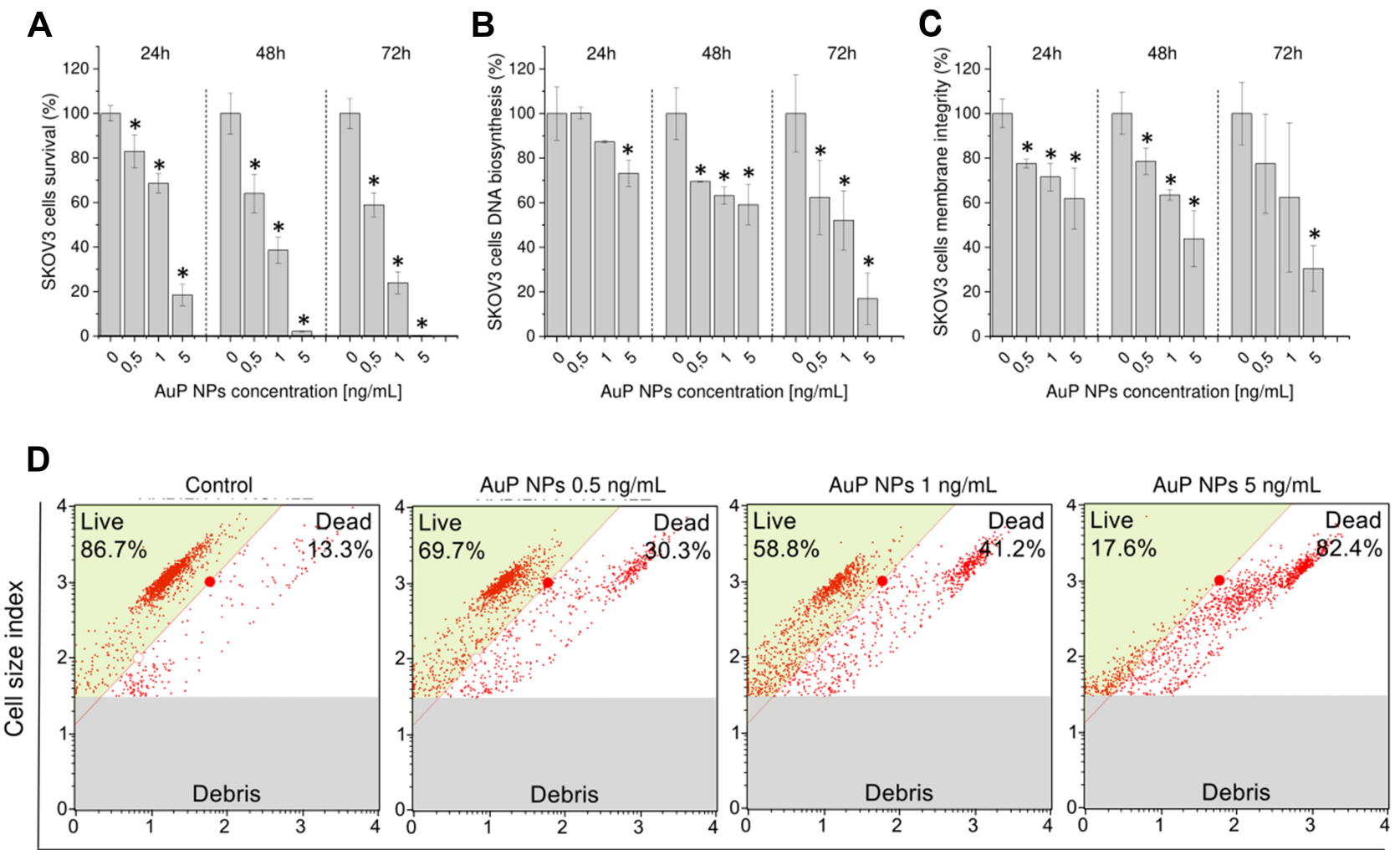

LIVE

Viability profile
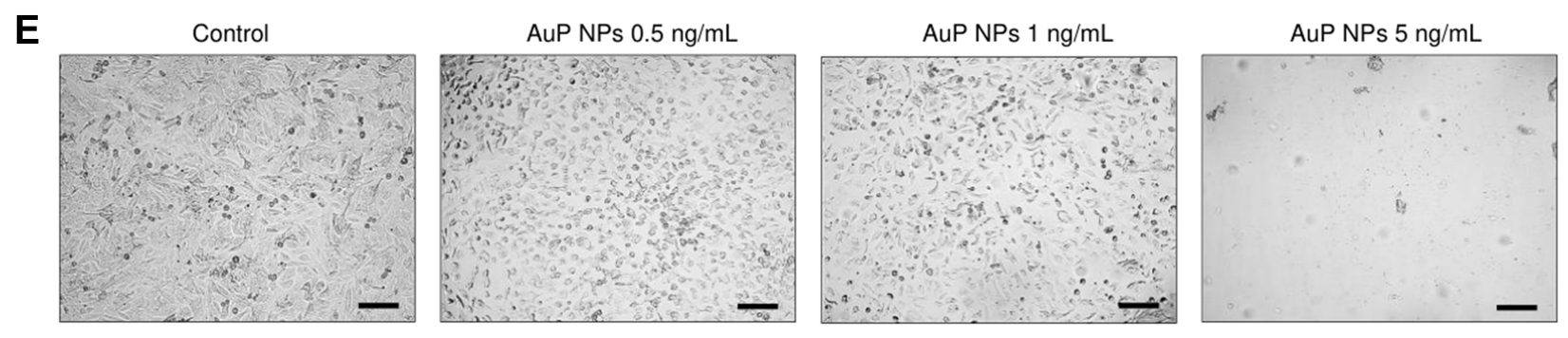

$\mathbf{F}$
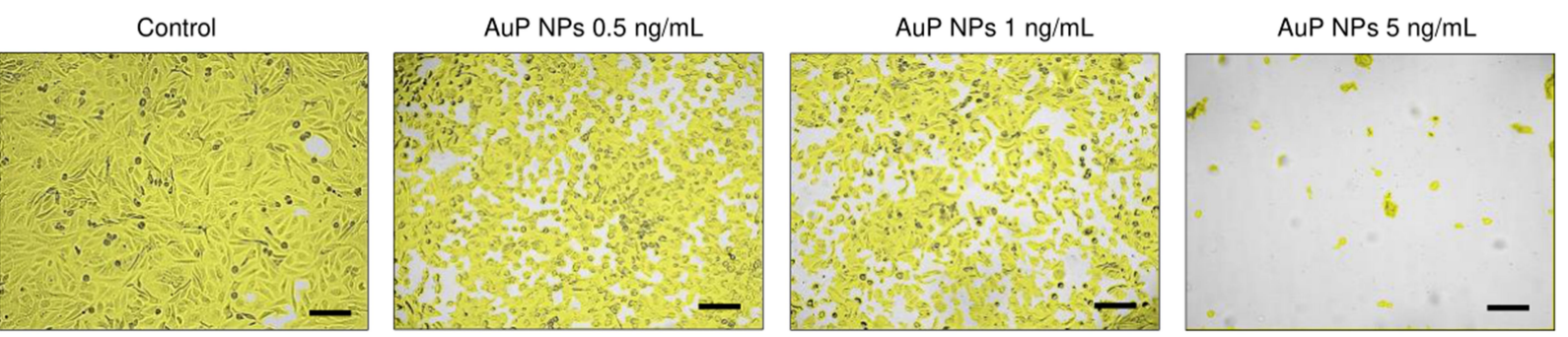

Figure 2 Cytotoxicity of peanut-shaped gold nanoparticles (AuP NPs) against human ovarian cancer SKOV3 cells. Decrease of cancer cell metabolic activity (A), DNA biosynthesis (B), and membrane permeability (C) upon treatment with peanut-shaped nanoparticles in doses ranging from $0.5-5 \mathrm{ng} / \mathrm{mL}$ for 24,48 , and 72 hours. Representative plots of flow-cytometry based analysis of cellular viability after 72 hour treatment of ovarian cells with peanut-shaped nanoparticles (D). Alterations in cellular morphology and confluence of cancer cells treated with AuP for 72 hours $(\mathbf{E}$ and $\mathbf{F}$, respectively). *Statistical significance $(P<0.05)$ when compared to untreated control cells. For panels $(\mathbf{A}-\mathbf{C})$ results from four independent experiments are shown. For panels $(\mathbf{D}, \mathbf{E}$, and $\mathbf{F})$ one representative result is shown.

The cytotoxic effects of gold nanoparticles were also analyzed using DNA biosynthesis assay. Accordingly, the proliferation capability of cells reflected by relative DNA content was reduced to $50.14 \pm 29.58 \%$ and $10.26 \pm 12.59 \%$ for AuP NPs at doses 1 and $5 \mathrm{ng} / \mathrm{mL}$ after 72 hours treatment (Figure 2B). Analysis of membrane permeability showed that gold nanoparticles significantly decreased the number of cells with intact membranes (Figure 2C). This observation was also confirmed using flow cytometry. Surprisingly, in contrast to MTT and DNA biosynthesis 
assay, demonstrating loss of metabolic activity and proliferation capability of SKOV-3 upon 72 hours treatment with $5 \mathrm{ng} / \mathrm{mL}$ of AuP NPs, approx. $25 \%$ of cells retain the integrity of cells membranes (Figure 2D). Importantly, comparative analysis of cytotoxic effects of developed nanopeanuts and spherical-shaped nanoparticles revealed that peanut-shaped gold nanoparticles are significantly more cytotoxic than spherical ones in comparable doses of nanomaterials. Although some effect of AuSph NPs on the biology of ovarian cancer SKOV-3 cells might be suggested, a majority of them do not reach statistical significance (Supplementary Figure 2). In comparison, AuP NPs strongly limit the metabolic activity of cancer cells, decrease DNA biosynthesis in SKOV-3 cells, and significantly affect membrane integrity, which strongly confirms our hypothesis that the non-spherical shape of gold nanopeanuts is a significant factor determining anticancer activity of AuP NPs against ovarian cancer cells.

Alterations of morphological features of AuP NPstreated SKOV-3 cells when compared to control were noted using a light-inverted microscope at a magnification of $\times 20$. As shown in Figure $2 \mathrm{E}$ and $\mathrm{F}$, untreated ovarian cancer cells grew adherently in culture plate, in $>95 \%$ confluency, and were characterized by regular size and shape. In contrast the cells treated with gold nanopeanuts, in dose-dependent response way, they lost the cellular shape and decline in cellular confluency. With increasing concentrations of AuP NPs, cells became rounded, start to detach from the cell culture surface, and loss in cell-cell contact was also recorded. When summarized, these results suggest that developed gold nanoparticles inhibit the growth of ovarian cancer cells in a dose- and time-dependent manner and this effect is the most prominent after 72 hours of treatment. Based on these preliminary results, 72 hours was selected as the appropriate incubation time in the following experiments.

\section{Gold Nanopeanuts Disrupt Oxidative Balance in Ovarian Cancer Cells}

To investigate whether gold nanoparticles induce reactive oxygen species formation and disrupt redox balance in treated cancer cells, oxidative stress-associated parameters were investigated. Initially, a level of free thiols, including reduced glutathione (GSH) was estimated. GSH being ROS-scavenger maintains intracellular redox stability in cells and is recognized as a key endogenous molecule in the antioxidant pathways. In effect, decline of its intracellular level introduces cells into excessive oxidative stress resulting in cell death. ${ }^{24}$ As demonstrated in Figure $3 \mathrm{~A}$ and $\mathrm{B}$, treatment of ovarian cancer cells with gold nanopeanuts interfered with GSSG-GSH balance leading to significant depletion of GSH and shifting of cellular cytosol from a reducing to an oxidizing environment. More quantitively, the number of cells with decreased GSH levels increased 3.7-fold when compared to untreated cells from $24.75 \pm 2.17 \%$ to $91.75 \pm 7.84 \%$ (for cells treated with AuP NPs at $5 \mathrm{ng} / \mathrm{mL}$ ) (Figure 3B).

In another set of experiments, AuP NPs-mediated upexpression of NADPH subunit 4 (NOX4) was confirmed using immunofluorescence staining (Figure 3C). A compelling number of studies confirm the crucial role of NOX4 in oxidative stress and present upregulation of its expression in cancer cells treated with ROS modulators. ${ }^{25,26}$ As a result of AuP NPs-mediated treatment, we recorded a significant increase of NADPH levels (Figure 3C), which encourage our hypothesis on disruption of redox balance in treated cancer cells. As expected, a significant overproduction of reactive oxygen species in AuR NPs-treated cells was recorded. When compared to control cells, ROS-derived fluorescence signal was increased $2.26 \pm 0.13,3.04 \pm 0.21$, and $7.11 \pm 0.62$-fold times after 24,48 , and 72 hours, respectively (Figure 3D), being in agreement with dose- and time-dependent effects of NPs-mediated treatment observed in cytotoxicity assays.

Finally, considering the reports indicating loss of mitochondrial potential in cells where GSH intracellular level drops below a threshold level, ${ }^{27}$ and some studies highlighting crosstalk between mitochondria and NADPH activity, ${ }^{28}$ we investigated whether AuP NPs-mediated treatment affect mitochondrial functioning. Loss of the mitochondrial membrane potential was detected using the lipophilic cationic dye JC-1 that displays potentialdependent accumulation in the mitochondria, thus analysis of JC-1-derived fluorescence signal allows for distinguishing between healthy and apoptotic cells. Our results indicate that the percentages of mitochondrial membrane depolarized cells was significantly increased upon exposition to AuP NPs at doses of $0.5,1$, and $5 \mathrm{ng} / \mathrm{mL}$ and were $31.67 \pm 6.93,76.33 \pm 13.01$, and $98.33 \pm 1.52 \%$, respectively, while the values recorded for the control was $24.00 \pm 6.93 \%$ (Figure 3E). The above results indicate that treatment of ovarian cancer cells is accompanied by disruption of the oxidative balance in cancer cells and impairment of protective anti-oxidative molecules, which subject cells into excessive oxidative stress and mitochondrial disfunction. 
A

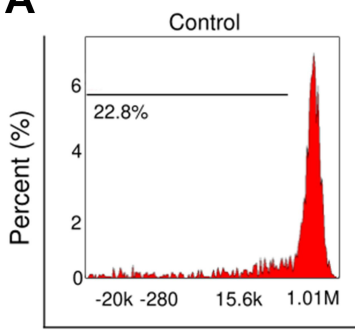

AuP NPs $0.5 \mathrm{ng} / \mathrm{mL}$

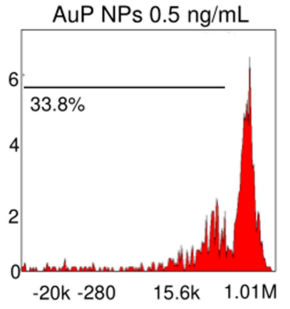

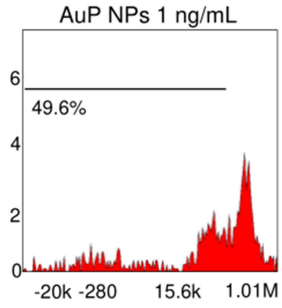

VB-48 Intensity
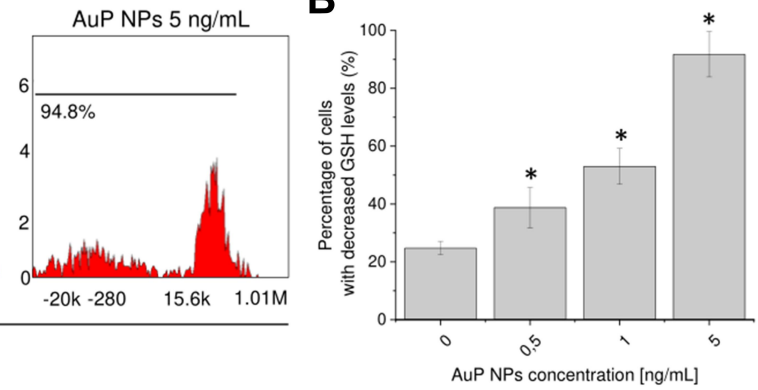

C

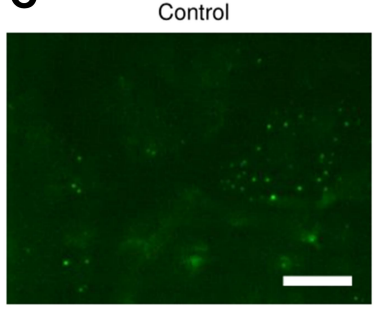

AuP NPs $0.5 \mathrm{ng} / \mathrm{mL}$

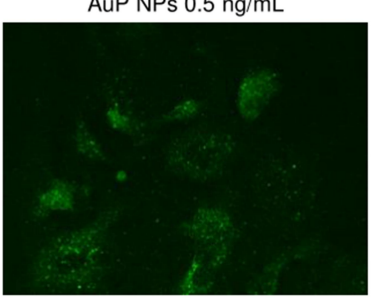

AuP NPs $1 \mathrm{ng} / \mathrm{mL}$

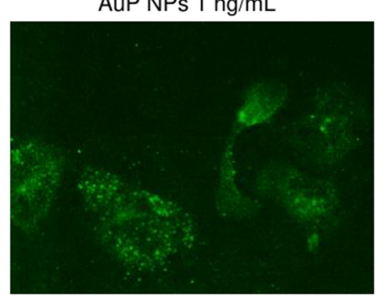

E
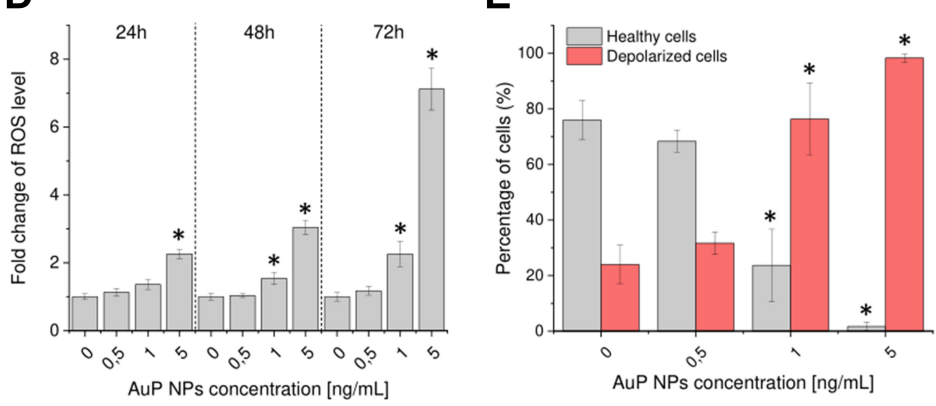

Figure 3 Disruption of oxidative balance in AuP NPs-treated ovarian cancer cells. Representative plots of flow-cytometry based analysis of GSH levels (A) and decrease of intracellular glutathione (GSH) concentration in treated cancer cells (B). Immunostaining of NADPH expression in AuP NPs-treated ovarian cancer cells (C). Induction of reactive oxygen species generation (D) and increase of number of cells with depolarized mitochondria (E) upon treatment with AuP NPs for 72 hours. For panels (A and $\mathbf{C}$ ) one representative experiment is shown. For panels $(\mathbf{B}, \mathbf{D}$, and $\mathbf{E})$ results are presented as mean \pm SD from three independent experiments. *Indicate statistical significance $(P<0.05)$ when compared to untreated control cells. Scale bar $\sim 10 \mu \mathrm{m}$.

\section{Developed Gold Nanopeanuts Activate JNK Signaling Pathway}

To more accurately investigate the molecular mechanism involved in observed killing activity, we investigated whether the JNK signaling pathway might be involved in this process. C-Jun N-terminal kinase (JNK) is recognized as an important member of the mitogen-activated protein kinase superfamily, activated by a number of environmental stimuli. Particularly, depletion of intracellular glutathione followed by generation of reactive oxygen species (ROS) and reactive nitrogen species (RNS) is required to activate this signaling pathway. ${ }^{29} \mathrm{ROS} / \mathrm{JNK}$ signaling is also reported to be implicated in the anticancer effect of a broad spectrum of antineoplastics. ${ }^{30-33}$ To confirm this assumption, we preincubated SKOV-3 cells for 2 hours with JNK inhibitor II (SP600125) before gold nanopeanuts addition. As demonstrated in Figure 4, some partial reverse of toxic effect of developed nanoparticles is observed upon addition of JNK inhibitor. By preincubation of SKOV-3 cells with JNK inhibitor II, statistically significant alterations in cell population distribution were noted when compared to samples treated with gold nanoparticles alone for 24 hours. When assessed using VB-48 $\mathrm{TM} /$ propidium iodide/orange acridine combined staining, we observed a rise of percentage of healthy cells with high viability (Figure 4A and B). Accordingly, a number of caspase-positive cells was also significantly diminished (Figure 4C and D), which confirms that the JNK signaling pathway is involved in AuP NPs-mediated biological activity. Importantly, activation of this pathway was also recorded to be important for further caspase activation and proceeding in the apoptosis process. 


\section{Gold Nanoparticles Regulate Apoptosis-Related Proteins}

Both decline in cellular glutathione concentration and dysregulation of the mitochondrial transmembrane potential are events occurring early on in apoptosis. ${ }^{34}$ Since both of these effects were observed in AuP NPs-treated samples, we concluded that anti-cancer activity of developed nanoparticles is related to apoptosis. To better explore how gold nanopeanuts affect this process, we characterized the alterations in selected apoptosis-related proteins expression upon AuP NPs exposure. Using immunofluorescence staining we elucidated whether some
A

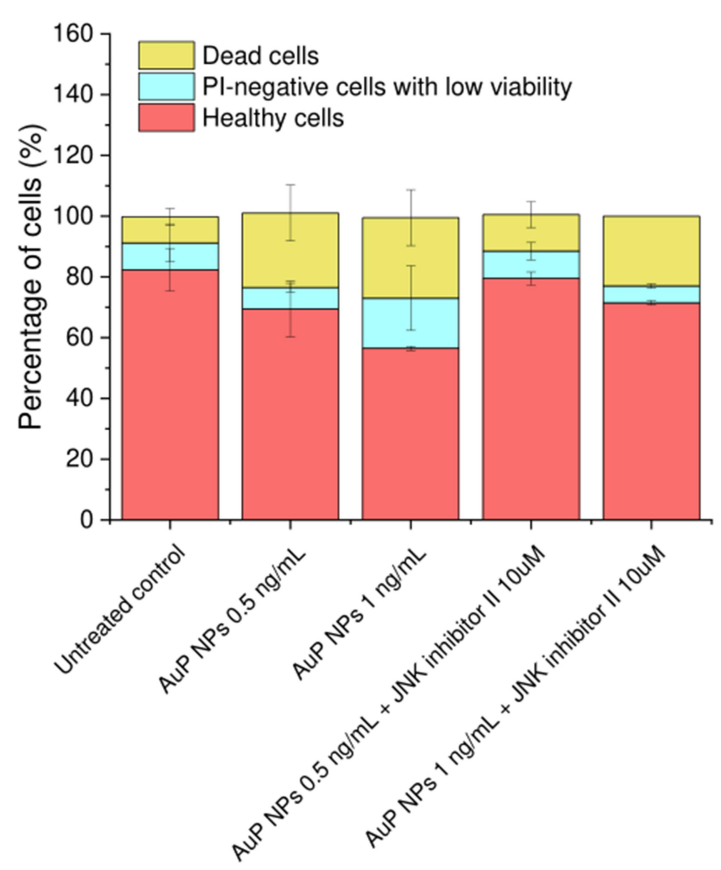

B

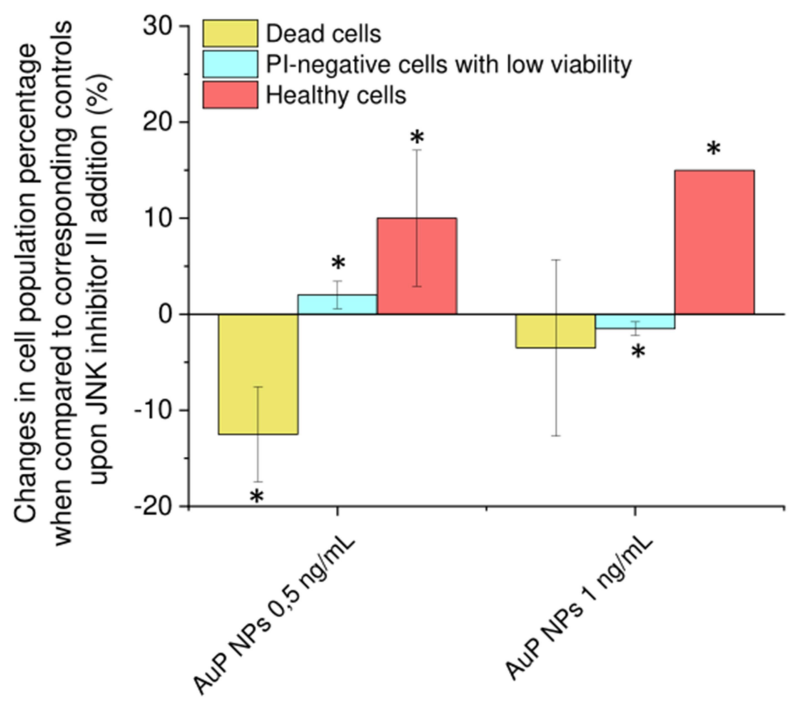

D

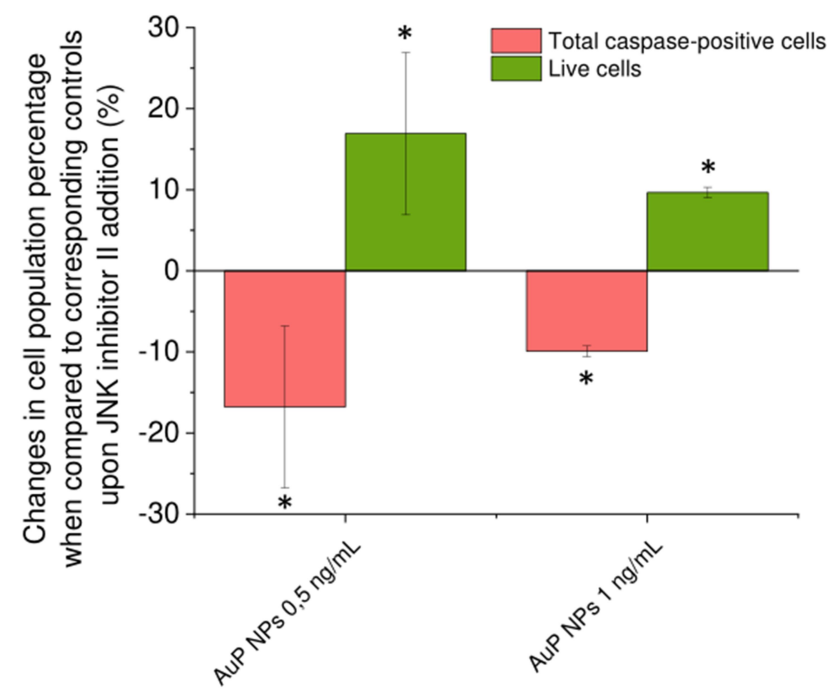

Figure 4 Involvement of JNK signaling pathway in AuP NPs-mediated killing. Distribution of cell viability; dead, PI-negative with low viability, and healthy cells (A) or dead, caspase-positive/dead, caspase-positive, and live cells (C) in samples of SKOV-3 cells treated with AuP NPs at doses of 0.5 and I ng/mL for 24 hours exclusively or preincubated with JNK inhibitor II prior to the addition of gold nanopeanuts. Relative alterations in cell population percentage upon JNK inhibitor II addition evaluated using Vitality assay $(\mathbf{B})$ or MultiCaspase Assay (D). *Statistical significance $(P<0.05)$ when compared to samples treated with AuP NPs without pre-incubation with inhibitor. 
alterations in functioning of phospholipid-binding proteins from annexins group, specifically annexin A1 (ANXA1), occur in AuP NPs-treated cells. Although ANXA1 was reported to be involved primarily in inflammation processes, it also has pro-apoptotic functions, which include activation of p38 and JNK signaling pathways, ${ }^{35}$ colocalization with phosphatidylserine on the outer plasma membrane leaflet, ${ }^{36}$ and activation of caspase-3. ${ }^{37}$ As demonstrated in Figure 5A, the signal from immunostained annexin A1 is highly increased in nanoparticlestreated cells, which confirm that treated ovarian cancer cells enter the apoptotic signaling pathway.

The apoptotic course of cell death, originating from the mitochondrial disbalance under oxidative stress, is regulated by apoptosis-related proteins, including Bcl-2. Specifically, Bcl-2 prevents apoptosis by either sequestering of caspases or by preventing the release into the cytoplasm of mitochondria-derived apoptogenic factors such as apoptosis-inducing factor (AIF) and cytochrome c. Thus, a decrease in Blc-2 activity promotes the apoptosis process and accelerates the cellular death. ${ }^{38}$ In our study, measurement of Bcl-2 activity after treatment was performed by flow cytometry. As shown in Figure 5B, activity of Bcl-2 protein when related to control cells was decreased by $75.65 \%$ when $5 \mathrm{ng} / \mathrm{mL}$ of AuP NPs was applied. Consequently, we observed a significant increase of caspases activity in treated cancer cells (Figure 5C). Combining the fluorescent-labeled inhibitor of caspases (FLICA) with 7-actinomycin D (membrane integrity indicator) allowed us to simultaneously measure caspase activation and cells death. Quantitively, the increase in number of live cells with activated caspases was not exceptional (rise from $5.15 \pm 0.91 \%$ for control cells to $18.77 \pm 4.77 \%$ for cells treated with $5 \mathrm{ng} / \mathrm{mL}$ of AuP NPs for 72 hours), but the percentage of dead, caspase-positive was $73.93 \pm 5.81 \%$ for AuP NPs $(5 \mathrm{ng} / \mathrm{mL})$ treated cells, which is nearly a 12 -fold increase when compared to control (Figure 5C). Since some caspases to not initiated in the apoptosis signal, but are rather involved in inflammatory cytokine signaling and other type of cell death, ${ }^{39}$ we decided additionally to verify the mechanisms of apoptosis of ovarian cancer cells induced by gold nanopeanuts using Western blot analysis of effectors caspases (caspase-3 and caspase-9), and PARP expression. As shown in Figure 5D and $\mathrm{E}$, gold nanopeanuts induced cleavage of caspase-3, caspase-9, and PARP in a dosedependent manner. Conversely, a significant depletion
$(73.52 \%)$ in cytochrome c oxidase expression was noted. The immunostaining analysis further corroborated these results. The above observations indicate that AuP NPs induced cell death in SKOV-3 cells by regulation of Bcl2 protein, depletion of COX IV and induction of caspasedependent apoptotic signaling.

\section{AuP NPs Promote Autophagy in SKOV-3 Cells}

To examine whether autophagy is induced in gold nanoparticles-treated ovarian cancer cells, we analyzed the expression of LC3A/B, beclin-1, Atg12, and Atg7, which are important autophagy-related proteins, using Western blot. As demonstrated in Figure 6, upon exposition to AuP NPs, expression of these protein increased dose-dependently. When compared to control cells, expression of LC3A/B and beclin-1 in AuP NPs-treated cells (5 ng/mL) increased 2.17- and 2.68-fold, while activity of Atg12 and Atg7 was higher by 2.86- and 4.25-times. This quantitative data were also reflected in immunofluorescence staining. Microphotographs demonstrated in Figure 6C confirm the upregulation of autophagic-related proteins by developed gold nanoparticles.

\section{Apoptosis and Autophagy Act Synergistically to Diminish Viability of Ovarian Cancer Cells}

To investigate the relationships between apoptosis and autophagy, we performed Western blot analysis using apoptosis (Z-VAD-FMK) and autophagy (3-methyladenine [3-MA]) inhibitors. As demonstrated in Figure 7 and in accordance with the above-presented data, developed gold nanoparticles induce cell growth suppression by triggering both autophagy and apoptosis in treated ovarian cancer cells. Consequently, the expression of several apoptosis marker (cleaved caspase-3, cleaved caspase-9, and cleaved-PARP), and autophagy (Atg7 and Beclin-1) is limited upon exposure to their respective inhibitors. These results confirm that AuP NPs induce these processes acting as an anti-cancer agent in SKOV-3 cells. In further analyses, aiming to explore either AuP NPs-induced autophagy promote cell survival or contribute to cell death, we concluded that both autophagy and apoptosis processes promote cell killing by synergistic mechanism. As demonstrated, autophagy inhibitor 3-methyladenine inhibits caspase activation and reduces apoptosis (panel B), and in similar manner - Pan-Caspase inhibitor Z-VAD-FMK reduces the expression of autophagic proteins (panel D) suggesting that they are induced in an independent 
A
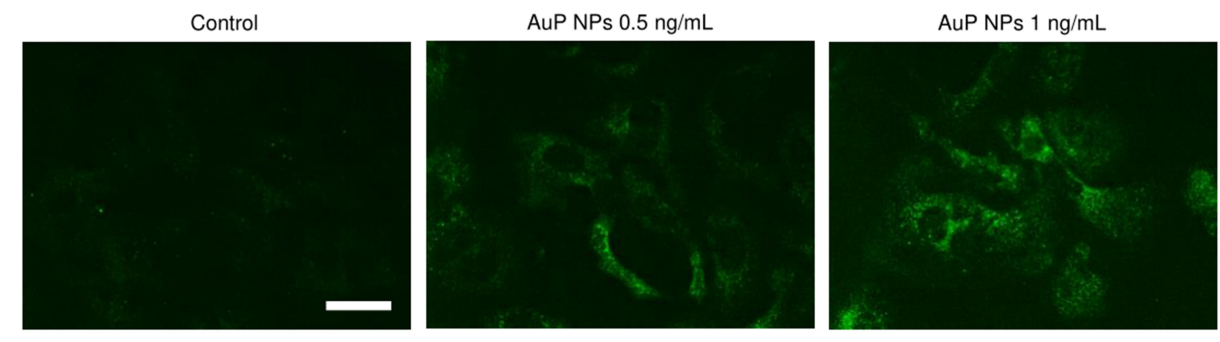

B

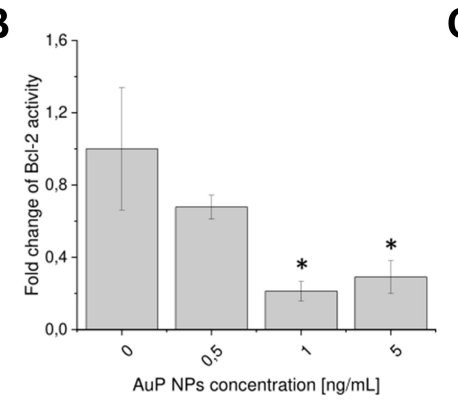

C

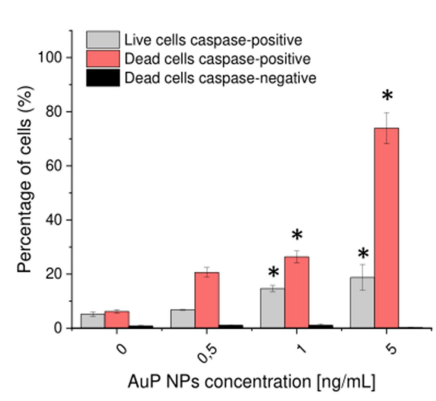

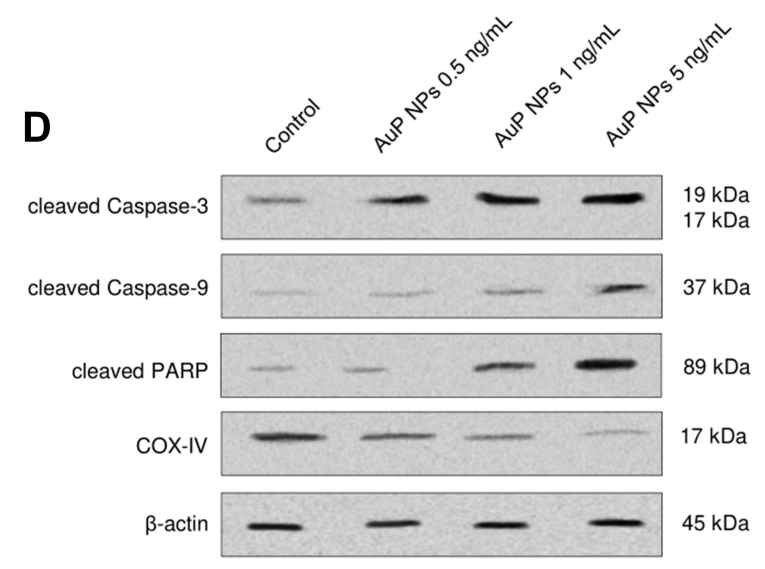

E

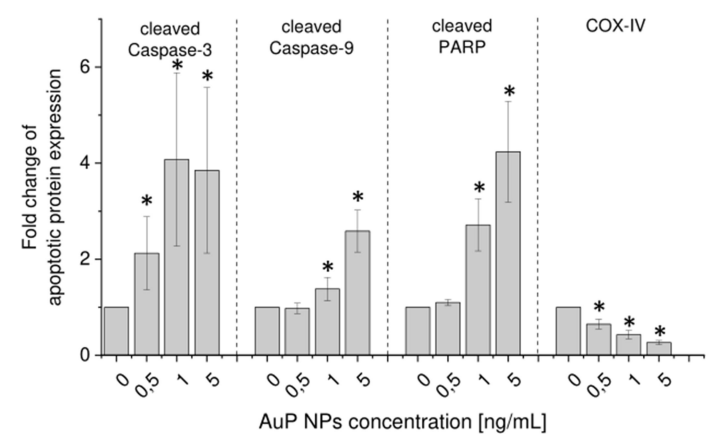

F

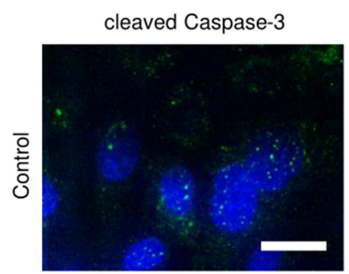

cleaved Caspase-9

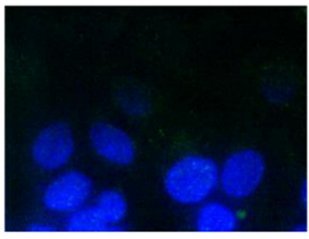

cleaved PARP
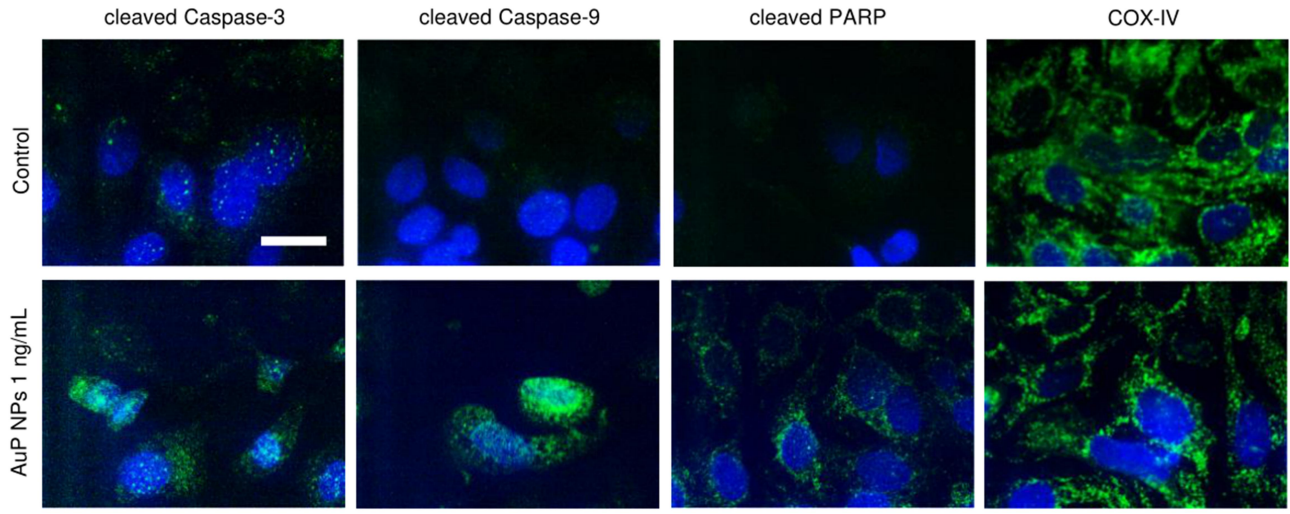

Figure 5 Mechanism of AuP NPs-induced apoptosis of ovarian cancer cells. Immunostaining of Annexin I (A), decrease of anti-apoptotic protein Blc-2 activity (B) and increase of number of caspase-positive cells (C) in AuP NPs-treated ovarian cancer cells. Western blot measurements of apoptotic proteins' expression: cleaved-Caspase-3, cleaved-Caspase-9, cleaved-PARP, and cytochrome C oxidase subunit 4 isoform I (COX-IV) in AuP NPs-treated cancer cells. Representative blots and densitometry analysis are presented in (D) and (E), respectively. (F) The immunofluorescence images of ovarian cancer cells treated with AuP NPs when compared to untreated controls. Blue color indicates fluorescence of Hoechst 33,342 - nucleic acid staining, while green color -FITC or -AlexaFluor $488-$ proteins staining. Scale bar $\sim 10 \mu \mathrm{m}$. For panels (A, D, and $\mathbf{F})$ representative results are presented. For panels $(\mathbf{B}, \mathbf{C}$, and $\mathbf{E})$ results are presented as mean \pm SD from three independent experiments. $*$ Statistical significance $(P<0.05)$ when compared to untreated control cells. 


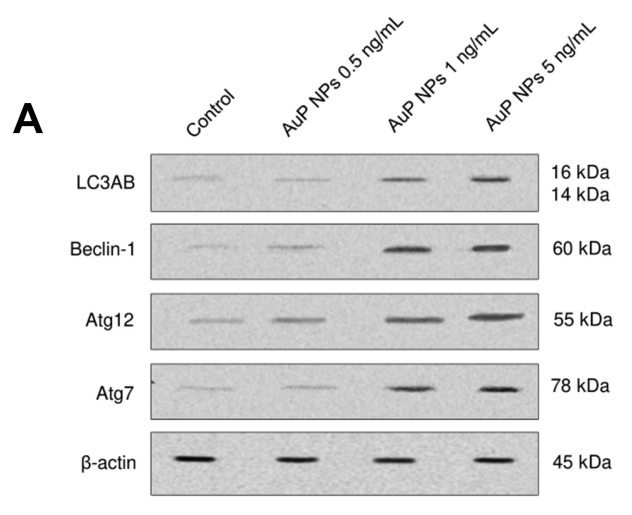

C
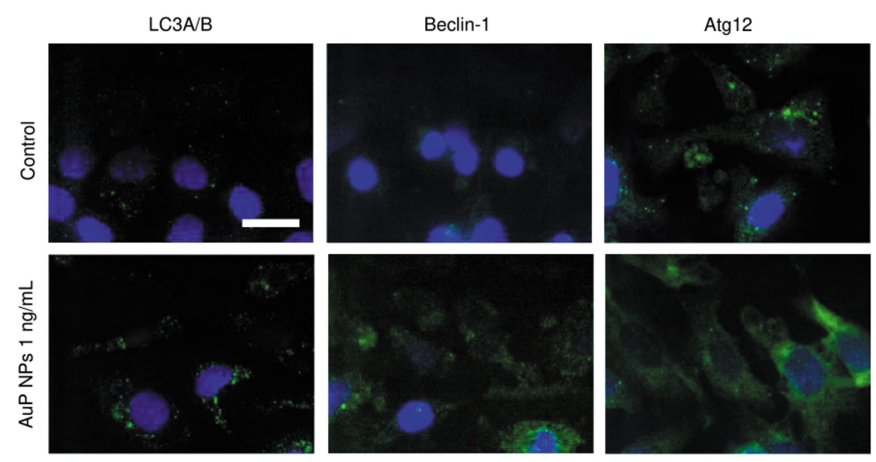

B

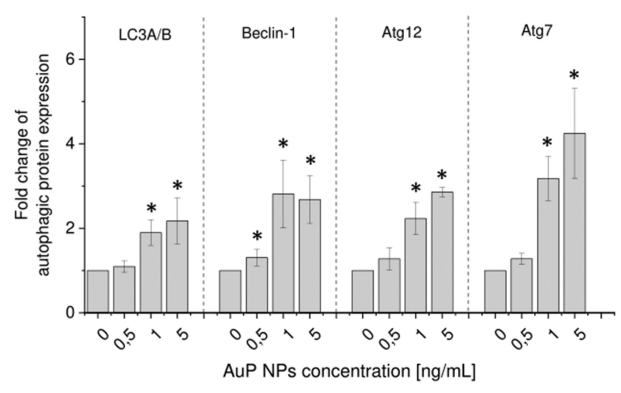

Figure 6 Induction of autophagy pathways in AuP NPs-treated ovarian cancer cells. Western blot measurements of expression of autophagic proteins: LC3A/B, Beclin-I, Atg12, and Atg7 in AuP NPs-treated cancer cells. Representative blots and densitometry analysis are presented in (A) and (B), respectively. (C) The immunofluorescence images of ovarian cancer cells treated with AuP NPs when compared to untreated controls. Blue color indicates fluorescence of Hoechst 33,342- nucleic acid staining, while green color -FITC or -AlexaFluor 488 - proteins staining. Scale bar $\sim 10 \mu \mathrm{m}$. For panels $(\mathbf{A}$ and $\mathbf{C})$ representative results are presented. For panel (B) results are presented as mean $\pm S D$ from three independent experiments. *Statistical significance $(P<0.05)$ when compared to untreated control cells.

manner and might work together in triggering cell death. In summary, the above data showed that gold nanopeanuts induced cell apoptosis and autophagy through the JNK signaling pathway, which is activated by reactive oxygen species and excessive oxidative stress resulting directly from a decrease of intracellular ROS scavengers.

\section{Discussion}

Owing to the plethora of unique physicochemical properties, including optical characteristic, high absorption efficiency, localized surface plasmon resonance (LSPR) effect, or extensive surface-to-volume ratio gold nanoparticles have been extensively explored for bioimaging and treatment of cancer. ${ }^{40}$ Importantly, highly optimized synthesis methods of gold nanoparticles allow specifically designed $\mathrm{Au}$ NPs with controllable surface chemistry, size and shape, thus morphological features that have a fundamental impact on their cellular uptake and cytotoxicity. ${ }^{11}$ Although some of the reports are contradictory, ${ }^{41,42}$ in general, non-spherical nanogolds are recognized as more cytotoxic than nanospheres with comparable size and dose. Most recently, Lee et $\mathrm{al}^{43}$ measured cytotoxicity of gold nanorods, gold nanostars, and gold nanospheres against four cancer cells types: stomach gastric cancer cells AGS, cervix adenocarcinoma cells HeLa, liver cancer cells HepG2, and colon cancer cells HT-29, and concluded that anti-cancer activity of such nanoparticles followed the order nanorods $>$ nanostars $>$ nanospheres. In another study, spherical and rod-shaped nanoparticles were reported to be more cytotoxic than nanoflowers or nanostars, which was determined primarily by their size and aggregation processes. ${ }^{44}$ With the intention to their application as improved anti-cancer agents, we developed elongated-type gold nanoparticles in the shape of nanopeanuts (Figure 1) and investigated their anti-cancer activity and mechanism of biological activity against ovarian cancer cells. According to our best knowledge, this is the first study describing the therapeutic 
A

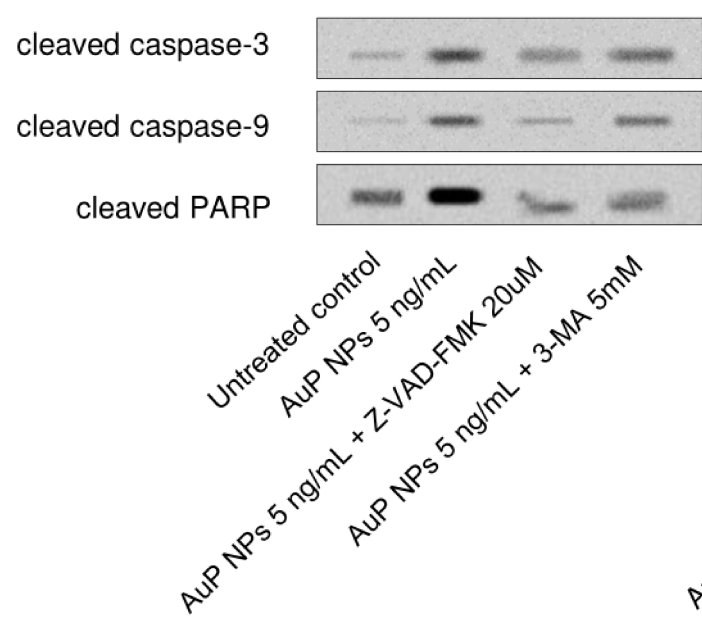

C
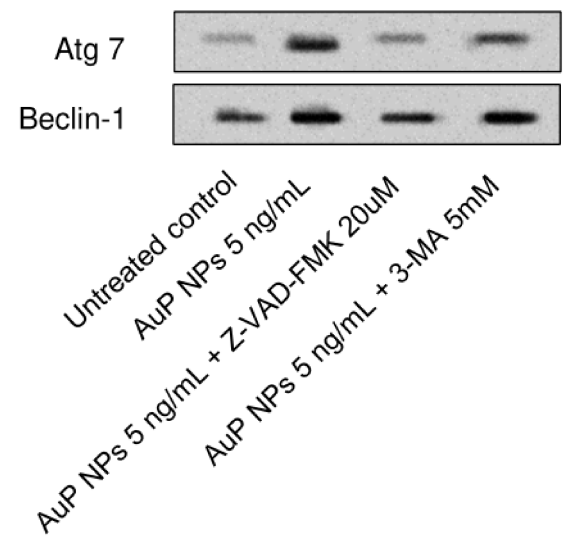

B

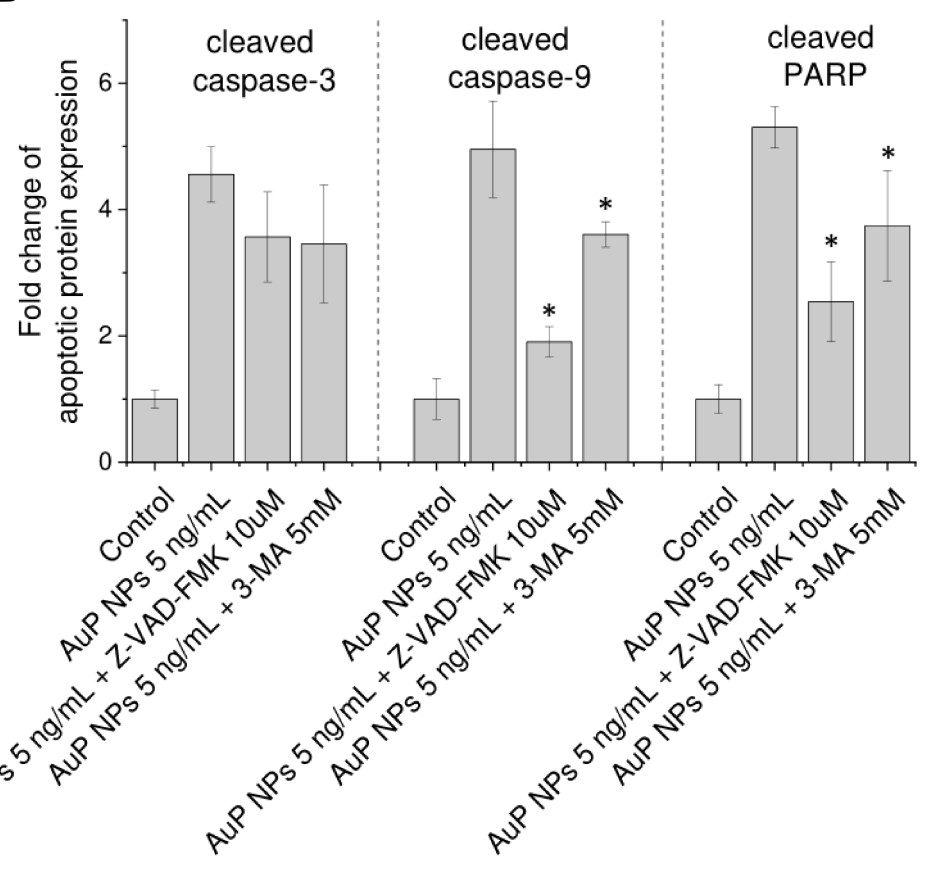

D

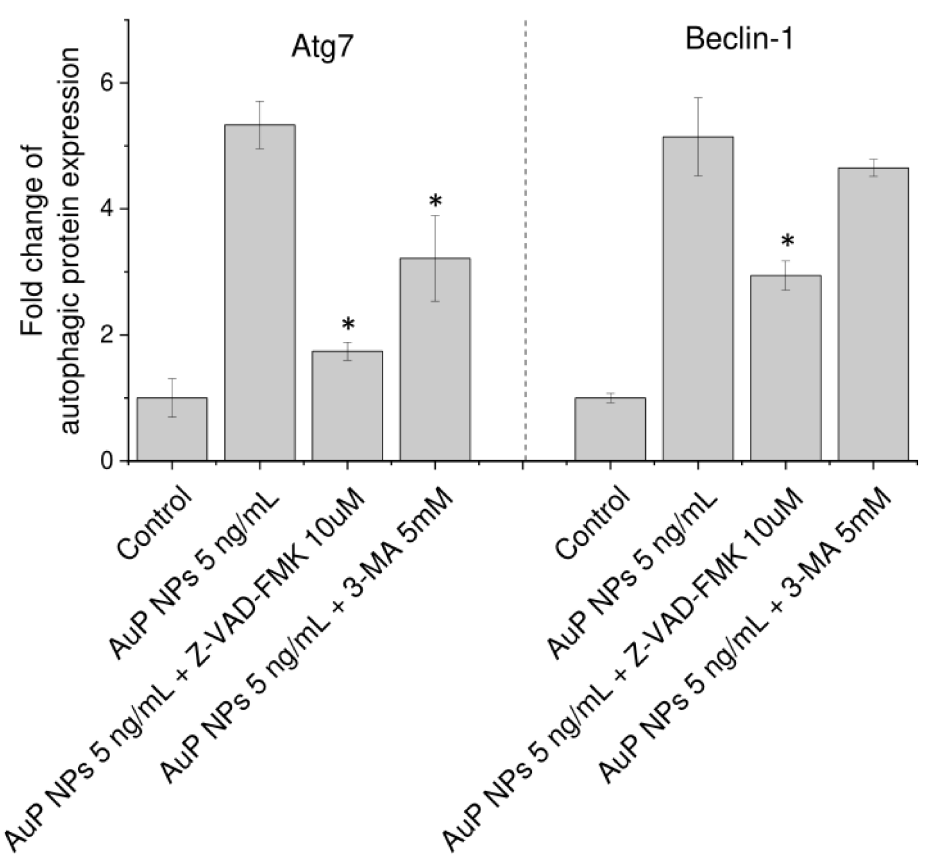

Figure 7 Western blot evaluation of apoptotic (A and B) and autophagic (C and D) proteins expression in human ovarian adenocarcinoma SKOV-3 cells upon treatment with peanut-shaped gold nanoparticles (AuP NPs) of $5 \mathrm{ng} / \mathrm{mL}$ for 24 hours. In this experiment, cells were pre-treated for 2 hours with signaling pathways inhibitors: apoptosis inhibitor (Z-VAD-FMK, $20 \mathrm{u \mu M}$ ), or autophagy inhibitor (3-MA, $5 \mathrm{mM})$. Results are presented as mean \pm SD from three independent experiments. *Statistical significance $(P<0.05)$ when compared to samples treated with AuP NPs without pre-incubation with inhibitor.

potential of peanut-shaped gold nanoparticles in the treatment of cancers. As demonstrated, synthesis modification and elongating the synthesis time up to 3 hours resulted in the generation of peanut-shaped gold nanoparticles with size $\sim 60 \pm 4.24 \mathrm{~nm}$ along the longitudinal axis, $\sim 30 \pm 3.49$ $\mathrm{nm}$ along the transverse axis and $\sim 25 \pm 3 \mathrm{~nm}$ in the narrowest place. Since a considerable drawback limiting clinical utility of gold nanoparticles is their low effectiveness in 
reaching a target diseased tissue (it is estimated that only $0.7 \%$ of initial nanoparticle dose is actually delivered to the tumors), it is crucial to design nanoparticles with improved cellular uptake ability. Peanut-shaped gold nanoparticles developed for the purpose of this study have a width-to-height ratio of 1:2 (Figure 1). Most recently, Tarantola et $\mathrm{al}^{45}$ revealed that rod-shaped gold nanoparticles with a lower width:height ratio are uptaken by mammalian cells with a higher degree than those with more elongated characteristics,which suggest that nanoparticles developed by us should be effectively internalized into cancer cells. In addition, trouble-free modification of the chemical structure of the obtained gold nanoparticles and the possibility of their conjugation with other anti-cancer agents also suggests the possibility of their use as drug transporters, in analogy to Au NPs-drug conjugates, as demonstrated previously. ${ }^{46}$ Nevertheless, this aspect needs confirmation in more complex in vivo models.

Cytotoxicity measurements indicated that peanutshaped gold nanoparticles are effective in doses as low as nanograms per milliliter. Accordingly, at concentration of $5 \mathrm{ng} / \mathrm{mL}$ only residual cancer cells survival is detected (Figure 2), which suggest their considerable anti-cancer potential. Importantly, developed gold nanopeanuts are far more effective than spherical-shaped gold nanoparticles (Supplementary Figure 2) and conventional cytostatics (Supplementary Table 1). Although peanut-shaped nanoparticles were not reported to date, an extensive research on other elongated-type gold nanoparticles, particularly gold nanorods, allow for some statements and hypothesis to be made. According to the results demonstrated in Figure 3, we state that gold nanopeanuts exert potent inhibitory activity by induction of excessive reactive oxygen species in treated cancer cells, which ultimately leads to disturbance of redox balance and introduction of ovarian cancer cells into oxidative stress. Reactive oxygen species, as highly reactive molecules derived from oxidative metabolism of cells, act as a second messenger in signaling pathways, thus have a pivotal role in regulating cell growth and differentiation, survival, or inflammation. ${ }^{47}$ At the same time, however, excessive ROS production results in irreversible oxidative damage, DNA destruction, and cell death via apoptosis, autophagy, or necroptosis pathways. ${ }^{48}$ To omit these cell-killing processes, a variety of anti-ROS protective mechanisms, including high levels of reduced GSH, was established in cells. ${ }^{24}$ We demonstrate that developed gold nanopeanuts effectively disturb these mechanisms by significant depletion of intracellular reduced glutathione level (Figure 3A and B). In agreement, previous studies confirmed that cytotoxicity of gold nanoparticles against lung cancer, breast cancer, ${ }^{49}$ and liver cancer cells ${ }^{50}$ is determined by loss of GSH and enrichment of cells with GSSH, resulting in impaired ability of cells to detoxify ROS. The mechanism of this AuP NPs-mediated reduction of GSH content upon exposure to tested nanoparticles might be explained by strong Au-S bonding interactions between $\mathrm{Au}$ NPs and GSH. ${ }^{51}$ Gold nanoparticles exert also considerably higher cytotoxicity in cells with artificially decreased GSH concentration, ${ }^{52}$ which additionally reaffirms our observations on ROS-mediated killing activity of gold nanopeanuts. To investigate the source of AuP NPsinduced reactive oxygen species, we investigated the expression of NOX4 in treated ovarian cancer cells (Figure 3C) and level of mitochondria depolarization in AuP NPs-treated cancer cells (Figure 3E). As demonstrated, a considerable upregulation of NOX4 expression and significant depolarization of mitochondria upon gold nanopeanuts treatment was noted. NADPH oxidase family members are recognized as one of intracellular ROS sources and their upregulation was reported to stimulate apoptosis and mitochondrial dysfunction in varied types of cells, ${ }^{53,54}$ which might suggest that AuP NPs-mediated cytotoxicity is NOX4-dependent. Nevertheless, available literature suggests that a more likely mechanism of anticancer activity of AuP NPs is through perturbations of the mitochondria than direct NOX4 activation. Compelling evidence indicates that mitochondria are a major target for NPs-induced oxidative stress and once nanoparticles gain access to mitochondria they initiate a pathway involving impairment of electron transport chain, structural damage, NADPH enzymes activation, and depolarization of mitochondrial membranes. ${ }^{55,56}$ Importantly, Sun et al ${ }^{56}$ demonstrated that physicochemical properties of gold nanoparticles, particularly surface charge, strongly determine the mechanism of oxidative stress induction. Accordingly, hydrophobic nanoparticles were reported to generate oxidative stress mainly via NOX activation while positively-charged NPs exerted their effect through perturbations of the mitochondria and modulation of intracellular calcium concentration. Consistently, cationic polystyrene nanospheres were noted to induce apoptosis by mitochondrial-targeting, as presented by Xia et al. ${ }^{55}$ Considering that gold nanopeanuts developed by our team were prepared using CTAB-assisted synthesis 
method and thus are positively-charged, we lean towards a theory that ROS-induction mechanism of AuP NPs involves disturbance in mitochondrial functioning, rather than direct NOX4 activation. Experiments SP600125 inhibitor confirmed also that AuP NPs act via JNK signaling pathway, ie, pathway activated mostly by ROS and RNS. ${ }^{29}$

As expected, mitochondrial disruption followed by ROS overproduction led to induction of cell killing processes (Figures 5-7). Apoptosis and autophagy, being type-I and type-II programmed cell death (PCD) processes, are recognized as a major cellular molecular mechanisms regulating cancer development and progression, and thus are utilized as potential targets for novel oncostatics. ${ }^{57,58}$ Apoptosis is characterized by specific morphological alterations and caspases activation, which leads ultimately to initiation of proteolytic processing cascades and elimination of damaged and unserviceable cells. ${ }^{58}$ Autophagy is an evolutionarily conserved major intracellular degradation mechanism by which aberrant proteins, damaged organelles, and other structures are digested in lysosomes and further recycled. In general, autophagy seems to play a protective role in the physiological and pathological cell processes and promote cell survival during various stress conditions. ${ }^{59}$ Nevertheless, accumulating findings evidenced that over-stimulation of autophagy above a certain level results in autophagic cell death coming from cellular self-consumption. ${ }^{59}$ In this work, we present that exposure to gold nanopeanuts results in induction of both apoptosis and autophagy processes, as evidenced by analysis of most crucial and best-recognized apoptotic and autophagic proteins (Figures 5-7). Coinduction of apoptosis and autophagy occurs often when cancer cells are treated with agents interfering with ROS balance. ${ }^{31,60}$ Notably, in this aspect the surface chemistry of developed nanoparticles was reported to be more important than shape. ${ }^{61,62}$ In agreement with our study, gold nanorods prepared using CTAB-assisted method were noted to induce apoptosis in breast and gastric adenocarcinoma ${ }^{61,63}$ and promote autophagy in a variety of cancer and non-cancerous tissues. ${ }^{62}$ These results were further confirmed in another study, demonstrating potent autophagy-induced abilities of CTAB-capped Au NPs in lung carcinoma cells. ${ }^{64}$ The above reports suggest that suitable decoration of gold nanoparticles may potentially be used in malignancies therapies. A question is left whether apoptosis and autophagy processes upon gold nanoparticles exposure inhibit or induce each other. Previous studies demonstrate that even when developed oncotherapeutics induce both of these processes, the ultimate cell fate is determined by the balance between anticancer apoptosis and pro-survival autophagy. ${ }^{31,60}$ Considerable cytotoxicity recorded for developed gold nanopeanuts strongly indicate that cell life balance is directed towards the eradication of onco-transformed cells. Indeed, based on results of Western blot analysis we concluded that both autophagy and apoptosis processes promote cell killing by synergistic mechanism (Figure 7).

Although results demonstrated here encourage the usefulness of gold nanopeanuts in the treatment of ovarian carcinoma it should be noted that they are only preliminary and require further confirmation in much complex in vivo models. A considerable limitation hampering the clinical introduction of these nanomaterials is their toxicity resulting from CTAB bilayer present on the surface of developed nanopeanuts. ${ }^{65}$ Since the amount of CTAB that left in AuP NPs solution after rinsing do not exceed 6\% (data not shown) and effective doses of AuP NPs against SKOV-3 are in range from $1-5 \mathrm{ng} / \mathrm{mL}$, we calculate that the amount of bound CTAB corresponds to 0.164-0.82 nM. Some studies indicate that such CTAB concentration might considerably affect cytotoxicity of gold nanoparticles, ${ }^{65}$ which is why we cannot exclude such possibility in our experimental settings. However, it should be noted that spherical-shaped gold nanoparticles also prepared using CTAB reagent are non-toxic for ovarian cancer cells (Supplementary Figure 2). In addition, according to our data, hemolytic activity of AuP NPs at doses of $1-5 \mathrm{ng} / \mathrm{mL}$ ranges from $1.37 \pm 0.03 \%$ to 12.58 $\pm 0.37 \%$ (data not shown).

Considering the sum of these data, it is justified to further explore the best administration route of these nanomaterials in animal models. Based on available literature, some assumptions can be made that the best route of administration of AuP NPs would be administrated directly into the tumor. Localized delivery of gold nanoparticles via intratumoral injection was proposed as an effective and safe approach to obtain the sufficient concentration of nanoparticles in targeted tumor without systemic side-effects on healthy tissues and to limit nonspecific biodistribution. ${ }^{66}$ To date, such drug delivery was used particularly for the purpose of nanoparticlebased radiosensitization, ${ }^{67}$ photothermal therapy ${ }^{68}$ and NPs-mediated co-treatment with conventional cytostatics, ${ }^{66}$ and for this reason the utility of this route of administration for AuP NPs application should also be considered. Nevertheless, no conclusion can be made 
without detailed in vivo studies. Interestingly, intratumoral injection provides also the possibility to utilize gold nanopeanuts as photothermal therapy agents, as demonstrated for other gold elongated-type nanoparticles. ${ }^{69}$ Our preliminary data confirm the potential of peanut-shaped gold nanoparticles as photosensitizers (Supplementary Figure 3). Moreover, the positions of UV-Vis peak, which is visible in revised Figure 1D clearly showed that AuP NPs are characterized by appropriate physicochemical properties to enable such application. As it is known, the strong interaction between the Au NPs and light locally generate heat in the nanoparticles, ${ }^{70}$ which caused the increase of temperature to over $43^{\circ} \mathrm{C}$. The high $\mathrm{Au}$ NPs solution temperature can be used in photothermal anticancer therapy, to damage and kill cancerous cells. ${ }^{71,72}$ In biomedical applications of $\mathrm{Au}$ NPs their Surface Plasmon Resonance (SPR) peak should be around 600-900 nm, because these wavelengths have small energy, which is safe for healthy cells. However, those aspectsneeds to be elucidated using an animal models setting.

\section{Conclusions}

In summary, the results of in vitro studies demonstrate that gold nanopeanuts can induce apoptosis and autophagy in SKOV-3 cells through ROS-mediated pathways and indicate that gold nanopeanuts, as newly-shaped gold nanomaterials, possess the great potential as nanotherapeutics. Further research aimed to elucidate the safety and detailed therapeutic potential of such nanoparticles in in vivo settings are justified and clinically relevant.

\section{Funding}

This work was financially supported by grants from the National Science Centre, Poland (UMO-2018/31/B/NZ6/ 02476 to RB), and Medical University of Bialystok (SUB/ 1/DN/20/003/1122 to EP). Part of the study was conducted with the use of equipment purchased by the Medical University of Białystok as part of the RPOWP 2007-2013 funding, Priority I, Axis 1.1, contract No. UDA- RPPD.01.01.00-20-001/15-00 dated June 26, 2015. This work was supported by the program of the Minister of Science and Higher Education under the name "Regional Initiative of Excellence in 2019-2022", project number: 024/RID/2018/19, financing amount: 11.999.000,00 PLN. The funders had no role in study design, data collection and analysis, decision to publish, or preparation of the manuscript.

\section{Disclosure}

JD and MP-W have a patent pending for synthesis and anti-cancer activity of gold nanopeanuts. The authors report no conflicts of interest for this work.

\section{References}

1. Desai A, Xu J, Aysola K, et al. Epithelial ovarian cancer: an overview. World J Transl Med. 2014;3(1):1-8. doi:10.5528/wjtm.v3.i1.1

2. McCreath WA, Chi DS. Surgical cytoreduction in ovarian cancer. Oncology. 2004;18(5):645-653.

3. Marchetti C, Pisano C, Facchini G, et al. First-line treatment of advanced ovarian cancer: current research and perspectives. Expert Rev Anticancer Ther. 2010;10(1):47-60. doi:10.1586/ era.09.167

4. Pokhriyal R, Hariprasad R, Kumar L, Hariprasad G. Chemotherapy resistance in advanced ovarian cancer patients. Biomark Cancer. 2019;11:1179299X19860815. doi:10.1177/1179299X19860815

5. Piktel E, Niemirowicz K, Wątek M, Wollny T, Deptuła P, Bucki R. Recent insights in nanotechnology-based drugs and formulations designed for effective anti-cancer therapy. J Nanobiotechnology. 2016;14(1):39. doi:10.1186/s12951-016-0193-x

6. Cabuzu D, Cirja A, Puiu R, Grumezescu AM. Biomedical applications of gold nanoparticles. Curr Top Med Chem. 2015;15 (16):1605-1613. doi:10.2174/1568026615666150414144750

7. Jain S, Hirst DG, O'Sullivan JM. Gold nanoparticles as novel agents for cancer therapy. $\mathrm{Br} J$ Radiol. 2012;85(1010):101-113. doi:10.1259/bjr/59448833

8. Kong FY, Zhang JW, Li RF, Wang ZX, Wang WJ, Wang W. Unique roles of gold nanoparticles in drug delivery, targeting and imaging applications. Molecules. 2017;22(9). doi:10.3390/ molecules22091445

9. Huang X, El-Sayed IH, Qian W, El-Sayed MA. Cancer cell imaging and photothermal therapy in the near-infrared region by using gold nanorods. J Am Chem Soc. 2006;128(6):2115-2120. doi:10.1021/ ja057254a

10. Dong YC, Hajfathalian M, Maidment PSN, et al. Effect of gold nanoparticle size on their properties as contrast agents for computed tomography. Sci Rep. 2019;9(1):14912. doi:10.1038/s41598-01950332-8

11. Kumar D, Mutreja I, Chitcholtan K, Sykes P. Cytotoxicity and cellular uptake of different sized gold nanoparticles in ovarian cancer cells. Nanotechnology. 2017;28(47):475101. doi:10.1088/1361-6528/ aa935e

12. Lee J, Chatterjee DK, Lee MH, Krishnan S. Gold nanoparticles in breast cancer treatment: promise and potential pitfalls. Cancer Lett. 2014;347(1):46-53. doi:10.1016/j.canlet.2014.02.006

13. Singh M, Harris-Birtill DC, Markar SR, Hanna GB, Elson DS. Application of gold nanoparticles for gastrointestinal cancer theranostics: a systematic review. Nanomedicine. 2015;11(8):2083-2098. doi:10.1016/j.nano.2015.05.010

14. Knights OB, McLaughlan JR. Gold nanorods for light-based lung cancer theranostics. Int J Mol Sci. 2018;19(11):3318. doi:10.3390/ ijms 19113318

15. Arvizo RR, Rana S, Miranda OR, Bhattacharya R, Rotello VM, Mukherjee P. Mechanism of anti-angiogenic property of gold nanoparticles: role of nanoparticle size and surface charge. Nanomedicine. 2011;7(5):580-587. doi:10.1016/j.nano.2011.01.011 
16. Qiao Y, Huang X, Nimmagadda S, et al. A robust approach to enhance tumor-selective accumulation of nanoparticles. Oncotarget. 2011;2(1-2):59-68. doi:10.18632/oncotarget.227

17. Nagayasu A, Uchiyama K, Kiwada H. The size of liposomes: a factor which affects their targeting efficiency to tumors and therapeutic activity of liposomal antitumor drugs. Adv Drug Deliv Rev. 1999;40 (1-2):75-87. doi:10.1016/s0169-409x(99)00041-1

18. Papahadjopoulos D, Allen TM, Gabizon A, et al. Sterically stabilized liposomes: improvements in pharmacokinetics and antitumor therapeutic efficacy. Proc Natl Acad Sci U S A. 1991;88(24):11460-11464. doi:10.1073/pnas.88.24.11460

19. Mayer LD, Tai LC, Ko DS, et al. Influence of vesicle size, lipid composition, and drug-to-lipid ratio on the biological activity of liposomal doxorubicin in mice. Cancer Res. 1989;49 (21):5922-5930.

20. Jain PK, Lee KS, El-Sayed IH, El-Sayed MA. Calculated absorption and scattering properties of gold nanoparticles of different size, shape, and composition: applications in biological imaging and biomedicine. $J$ Phys Chem B. 2006;110(14):7238-7248. doi:10.1021/jp057170o

21. Piktel E, Prokop I, Wnorowska U, et al. Ceragenin CSA-13 as free molecules and attached to magnetic nanoparticle surfaces induce caspase-dependent apoptosis in human breast cancer cells via disruption of cell oxidative balance. Oncotarget. 2018;9(31):21904-21920. doi:10.18632/oncotarget.25105

22. Borenfreund E, Puerner JA. Toxicity determined in vitro by morphological alterations and neutral red absorption. Toxicol Lett. 1985;24 (2-3):119-124. doi:10.1016/0378-4274(85)90046-3

23. Laemmli UK. Cleavage of structural proteins during the assembly of the head of bacteriophage T4. Nature. 1970;227(5259):680-685. doi: $10.1038 / 227680 \mathrm{a} 0$

24. Lv H, Zhen C, Liu J, Yang P, Hu L, Shang P. Unraveling the potential role of glutathione in multiple forms of cell death in cancer therapy. Oxid Med Cell Longev. 2019;2019:3150145. doi:10.1155/2019/ 3150145

25. Seo SU, Kim TH, Kim DE, Min KJ, Kwon TK. NOX4-mediated ROS production induces apoptotic cell death via down-regulation of c-FLIP and Mcl-1 expression in combined treatment with thioridazine and curcumin. Redox Biol. 2017;13:608-622. doi:10.1016/j. redox.2017.07.017

26. Zhu P, Tong BM, Wang R, et al. Nox4-dependent ROS modulation by amino endoperoxides to induce apoptosis in cancer cells. Cell Death Dis. 2013;4(3):e552. doi:10.1038/cddis.2013.68

27. Di Stefano A, Frosali S, Leonini A, et al. GSH depletion, protein S-glutathionylation and mitochondrial transmembrane potential hyperpolarization are early events in initiation of cell death induced by a mixture of isothiazolinones in HL60 cells. Biochim Biophys Acta. 2006;1763(2):214-225. doi:10.1016/j.bbamcr.2005.12.012

28. Dikalov S. Cross talk between mitochondria and NADPH oxidases. Free Radic Biol Med. 2011;51(7):1289-1301. doi:10.1016/j. freeradbiomed.2011.06.033

29. Shen HM, Liu ZG. JNK signaling pathway is a key modulator in cell death mediated by reactive oxygen and nitrogen species. Free Radic Biol Med. 2006;40(6):928-939. doi:10.1016/j. freeradbiomed.2005.10.056

30. Han J, Lv W, Sheng H, et al. Ecliptasaponin A induces apoptosis through the activation of ASK $1 / \mathrm{JNK}$ pathway and autophagy in human lung cancer cells. Ann Transl Med. 2019;7(20):539. doi:10.21037/atm.2019.10.07

31. Zhao Q, Liu Y, Zhong J, et al. Pristimerin induces apoptosis and autophagy via activation of ROS/ASK1/JNK pathway in human breast cancer in vitro and in vivo. Cell Death Discov. 2019;5 (1):125. doi:10.1038/s41420-019-0208-0

32. Zhao S, Fan S, Shi Y, et al. Propranolol induced apoptosis and autophagy. J Cancer. 2020;11(20):5900-5910. doi:10.7150/jca.46556
33. Wang $\mathrm{S}, \mathrm{Li} \mathrm{H}$, Chen $\mathrm{S}$, et al. Andrographolide induces apoptosis in human osteosarcoma cells via the ROS/JNK pathway. Int $J$ Oncol. 2020;56(6):1417-1428. doi:10.3892/ijo.2020.5032

34. Elmore S. Apoptosis: a review of programmed cell death. Toxicol Pathol. 2007;35(4):495-516. doi:10.1080/01926230701320337

35. Hsiang CH, Tunoda T, Whang YE, Tyson DR, Ornstein DK. The impact of altered annexin I protein levels on apoptosis and signal transduction pathways in prostate cancer cells. Prostate. 2006;66 (13):1413-1424. doi:10.1002/pros.20457

36. Arur S, Uche UE, Rezaul K, et al. Annexin I is an endogenous ligand that mediates apoptotic cell engulfment. Dev Cell. 2003;4 (4):587-598. doi:10.1016/s1534-5807(03)00090-x

37. Solito E, de Coupade C, Canaider S, Goulding NJ, Perretti M. Transfection of annexin 1 in monocytic cells produces a high degree of spontaneous and stimulated apoptosis associated with caspase-3 activation. $\mathrm{Br}$ J Pharmacol. 2001;133(2):217-228. doi:10.1038/sj.bjp.0704054

38. Edlich F. BCL-2 proteins and apoptosis: recent insights and unknowns. Biochem Biophys Res Commun. 2018;500(1):26-34. doi:10.1016/j.bbrc.2017.06.190

39. Li J, Yuan J. Caspases in apoptosis and beyond. Oncogene. 2008;27 (48):6194-6206. doi:10.1038/onc.2008.297

40. Singh P, Pandit S, Mokkapati VRSS, Garg A, Ravikumar V, Mijakovic I. Gold nanoparticles in diagnostics and therapeutics for human cancer. Int J Mol Sci. 2018;19(7):Jul. doi:10.3390/ ijms 19071979

41. Favi PM, Gao M, Johana Sepúlveda Arango L. Shape and surface effects on the cytotoxicity of nanoparticles: gold nanospheres versus gold nanostars. J Biomed Mater Res A. 2015;103(11):3449-3462. doi:10.1002/jbm.a.35491

42. Tarantola M, Pietuch A, Schneider D, et al. Toxicity of gold-nanoparticles: synergistic effects of shape and surface functionalization on micromotility of epithelial cells. Nanotoxicology. 2011;5(2):254-268. doi:10.3109/17435390.2010.528847

43. Lee YJ, Ahn EY, Park Y. Shape-dependent cytotoxicity and cellular uptake of gold nanoparticles synthesized using green tea extract. Nanoscale Res Lett. 2019;14(1):129. doi:10.1186/s11671-019-2967-1

44. Woźniak A, Malankowska A, Nowaczyk G, et al. Size and shape-dependent cytotoxicity profile of gold nanoparticles for biomedical applications. J Mater Sci Mater Med. 2017;28(6):92. doi:10.1007/s10856-017-5902-y

45. Chithrani BD, Ghazani AA, Chan WC. Determining the size and shape dependence of gold nanoparticle uptake into mammalian cells. Nano Lett. 2006;6(4):662-668. doi:10.1021/n1052396o

46. Chen J, Li X, Zhao X, et al. Doxorubicin-conjugated pH-responsive gold nanorods for combined photothermal therapy and chemotherapy of cancer. Bioact Mater. 2018;3(3):347-354. doi:10.1016/j. bioactmat.2018.05.003

47. Forman HJ, Maiorino M, Ursini F. Signaling functions of reactive oxygen species. Biochemistry. 2010;49(5):835-842. doi:10.1021/ bi9020378

48. Redza-Dutordoir M, Averill-Bates DA. Activation of apoptosis signalling pathways by reactive oxygen species. Biochim Biophys Acta. 2016;1863(12):2977-2992. doi:10.1016/j.bbamcr.2016.09.012

49. Mukherjee S, Dasari M, Priyamvada S, Kotcherlakota R, Bollu VS, Patra CR. A green chemistry approach for the synthesis of gold nanoconjugates that induce the inhibition of cancer cell proliferation through induction of oxidative stress and their in vivo toxicity study. J Mater Chem B. 2015;3(18):3820-3830. doi:10.1039/c5tb00244c

50. De Araújo RF, Pessoa JB, Cruz LJ, et al. Apoptosis in human liver carcinoma caused by gold nanoparticles in combination with carvedilol is mediated via modulation of MAPK/Akt/mTOR pathway and EGFR/FAAD proteins. Int $J$ Oncol. 2018;52(1):189-200. doi:10.3892/ijo.2017.4179

51. Chen SJ, Chang HT. Nile red-adsorbed gold nanoparticles for selective determination of thiols based on energy transfer and aggregation. Anal Chem. 2004;76(13):3727-3734. doi:10.1021/ac049787s 
52. Zhao Y, Gu X, Ma H, He X, Liu M, Ding Y. Association of glutathione level and cytotoxicity of gold nanoparticles in lung cancer cells. J Phys Chem C. 2011;115(26):12797-12802. doi:10.1021/jp2025413

53. Graham KA, Kulawiec M, Owens KM, et al. NADPH oxidase 4 is an oncoprotein localized to mitochondria. Cancer Biol Ther. 2010;10 (3):223-231. doi:10.4161/cbt.10.3.12207

54. Ago T, Kuroda J, Pain J, Fu C, Li H, Sadoshima J. Upregulation of Nox4 by hypertrophic stimuli promotes apoptosis and mitochondrial dysfunction in cardiac myocytes. Circ Res. 2010;106(7):1253-1264. doi:10.1161/CIRCRESAHA.109.213116

55. Xia T, Kovochich M, Brant J, et al. Comparison of the abilities of ambient and manufactured nanoparticles to induce cellular toxicity according to an oxidative stress paradigm. Nano Lett. 2006;6 (8):1794-1807. doi:10.1021/n1061025k

56. Sun H, Liu Y, Bai X, et al. Induction of oxidative stress and sensitization of cancer cells to paclitaxel by gold nanoparticles with different charge densities and hydrophobicities. J Mater Chem B. 2018;6(11):1633-1639. doi:10.1039/c7tb03153j

57. Fan YJ, Zong WX. The cellular decision between apoptosis and autophagy. Chin J Cancer. 2013;32(3):121-129. doi:10.5732/ cjc.012.10106

58. Melet A, Song K, Bucur O, Jagani Z, Grassian AR, Khosravi-Far R. Apoptotic pathways in tumor progression and therapy. Adv Exp Med Biol. 2008;615:47-79. doi:10.1007/978-1-4020-6554-5 4

59. White E, DiPaola RS. The double-edged sword of autophagy modulation in cancer. Clin Cancer Res. 2009;15(17):5308-5316. doi:10.1158/1078-0432.CCR-07-5023

60. Xu J, Zhang G, Tong Y, Yuan J, Li Y, Song G. Corilagin induces apoptosis, autophagy and ROS generation in gastric cancer cells in vitro. Int $\mathrm{J} \mathrm{Mol}$ Med. 2019;43(2):967-979. doi:10.3892/ijmm.2018.4031

61. Zhang F, Zhu X, Gong J, et al. Lysosome-mitochondria-mediated apoptosis specifically evoked in cancer cells induced by gold nanorods. Nanomedicine (Lond). 2016;11(15):1993-2006. doi:10.2217/ nnm-2016-0139

62. Wan J, Wang JH, Liu T, Xie Z, Yu XF, Li W. Surface chemistry but not aspect ratio mediates the biological toxicity of gold nanorods in vitro and in vivo. Sci Rep. 2015;5(1):11398. doi:10.1038/ srep 11398
63. Bhamidipati M, Fabris L. Multiparametric assessment of gold nanoparticle cytotoxicity in cancerous and healthy cells: the role of size, shape, and surface chemistry. Bioconjug Chem. 2017;28(2):449-460. doi:10.1021/acs.bioconjchem.6b00605

64. Li S, Zhang C, Cao W, et al. Anchoring effects of surface chemistry on gold nanorods: modulating autophagy. J Mater Chem B. 2015;3 (16):3324-3330. doi:10.1039/C5TB00076A

65. Alkilany AM, Nagaria PK, Hexel CR, Shaw TJ, Murphy CJ, Wyatt MD. Cellular uptake and cytotoxicity of gold nanorods: molecular origin of cytotoxicity and surface effects. Small. 2009;5 (6):701-708. doi:10.1002/smll.200801546

66. Zhang X, Teodoro JG, Nadeau JL. Intratumoral gold-doxorubicin is effective in treating melanoma in mice. Nanomedicine. 2015;11 (6):1365-1375. doi:10.1016/j.nano.2015.04.001

67. Boateng F, Ngwa W. Delivery of nanoparticle-based radiosensitizers for radiotherapy applications. Int J Mol Sci. 2019;21(1):273. doi:10.3390/ijms21010273

68. Kennedy LC, Bickford LR, Lewinski NA, et al. A new era for cancer treatment: gold-nanoparticle-mediated thermal therapies. Small. 2011;7(2):169-183. doi:10.1002/smll.201000134

69. Mooney R, Roma L, Zhao D, et al. Neural stem cell-mediated intratumoral delivery of gold nanorods improves photothermal therapy. ACS Nano. 2014;8(12):12450-12460. doi:10.1021/ $\mathrm{nn} 505147 \mathrm{w}$

70. Govorova AO, Richardson HH. Generating heat with metal nanoparticles. NanoToday. 2007;2(1):30-38. doi:10.1016/S17480132(07)70017-8

71. Yun SH, Kwok SJJ. Light in diagnosis, therapy and surgery. Nat Biomed Eng. 2017. doi:10.1038/s41551-016-0008

72. Liu Y, Yang M, Zhang J, et al. Human induced pluripotent stem cells for tumor targeted delivery of gold nanorods and enhanced photothermal therapy. ACS Nano. 2016;10(2):2375-2385. doi:10.1021/ acsnano. $5 \mathrm{~b} 07172$
International Journal of Nanomedicine

\section{Publish your work in this journal}

The International Journal of Nanomedicine is an international, peerreviewed journal focusing on the application of nanotechnology in diagnostics, therapeutics, and drug delivery systems throughout the biomedical field. This journal is indexed on PubMed Central, MedLine, CAS, SciSearch ${ }^{\circledR}$, Current Contents ${ }^{\circledR} /$ Clinical Medicine,

\section{Dovepress}

Journal Citation Reports/Science Edition, EMBase, Scopus and the Elsevier Bibliographic databases. The manuscript management system is completely online and includes a very quick and fair peer-review system, which is all easy to use. Visit http://www.dovepress.com/ testimonials.php to read real quotes from published authors. 